\title{
Regions of rationality: Maps for bounded agents
}

\author{
Robin M. Hogarth \& Natalia Karelaia* \\ Universitat Pompeu Fabra, Barcelona
}

\begin{abstract}
An important problem faced by boundedly rational agents is to identify "regions of rationality," i.e., the areas for which simple, boundedly rational models are and are not effective. To map the contours of such regions, we derive probabilities that models identify the best of $m$ alternatives $(m \geq 2)$ characterized by $k$ attributes $(k \geq 1)$. The models include a single variable (lexicographic), variations of elimination-by-aspects, equal weighting, hybrids of the preceding, and models exploiting dominance. We compare all with multiple regression. We illustrate the theory with twenty simulated and four empirical datasets. Fits between predictions and realizations are excellent. However, the terrain mapped by our work is complex and no single model is "best." We further provide an overview by regressing the performance of the different models on factors characterizing environments. We conclude with suggestions for further research as well as implications for the concept of rationality in economics.
\end{abstract}

Keywords: Decision making, Bounded rationality, Lexicographic rules, Choice theory

April 2005

* Robin M. Hogarth is ICREA Research Professor, and Natalia Karelaia is a graduate student at Universitat Pompeu Fabra, Barcelona, Spain. The authors are grateful for feedback received at workshops at the Center for Decision Research at the University of Chicago and Universitat Pompeu Fabra. This research was financed partially by a grant from the Spanish Ministerio de Ciencia y Tecnología.

For correspondence, please contact Robin M. Hogarth at Universitat Pompeu Fabra, Department of Economics and Business, Ramon Trias Fargas 25-27, 08005, Barcelona, Spain. (Tel: +34 93542 2561, Fax + 3493542 1746). Email: robin.hogarth@upf.edu, natalia.karelaia@upf.edu 
In his autobiography, Herbert Simon (1991) used the metaphor of a maze to characterize a person's life. In this metaphor, people are continually faced by choices involving two or more alternatives, the outcomes of which cannot be perfectly predicted from the information available prior to choosing. ${ }^{1}$ Extending this metaphor, the maze of choices faced by an economic agent can be thought of as a journey that crosses different regions varying in the types of questions posed.

If endowed with unbounded rationality, an agent could simply calculate the optimal responses to all questions. However, following Simon's insights, the bounded nature of the agent's rational capacities necessarily leads to following satisficing decision rules. Fortunately, satisficing does not imply unsatisfactory outcomes if the agent recognizes and implements the type of response appropriate to the region in which choice is being exercised.

The maze of choices faced by an agent can therefore be conceptualized as involving different "regions of rationality" where success depends on identifying decision rules that are appropriate to each region. In some regions, for example, the simplest random choice mechanism might be sufficient (e.g., choosing a lottery ticket). In other regions, returns to computationally demanding algorithms are potentially important (e.g., planning production in an oil refinery). What unbounded agents require therefore is knowledge - or maps - that indicate the demand for rationality in different regions. In particular, since attention is the scarce resource (Simon, 1978), it is critical that agents know what and how much information they should seek to make decisions in different regions.

The purpose of this paper is to contribute to defining maps that characterize regions of rationality for common problems faced by boundedly rational agents.

\footnotetext{
${ }^{1}$ As Simon (1991) points out, this metaphor also underlies his classic (1956) paper on what an organism needs to be able to choose effectively in given environments.
} 
Specifically, we consider choice points where agents must select one of two or more alternatives based on information that is probabilistically related to the criterion of choice. The structure of these tasks can be conceptualized as involving either multiple-cue prediction or multi-attribute choice and, as such, is common. In all cases, we construct theoretical models that predict the effectiveness in different regions of several, simple choice rules - or heuristics (see below) - thereby mapping the contours in which the various models are more or less successful.

Recently, the economic literature has shown increasing interest in models of bounded rationality (see, e.g., Conlisk, 1996; Camerer, 1998; Starmer, 2000; Kahneman, 2003). In addition to the influence of Simon, much interest was stimulated by research on heuristics and biases (Kahneman, Slovic, \& Tversky, 1982) that demonstrated how simple (or less than fully rational) processes produce outcomes that deviate from normative prescriptions. In addition, many papers in behavioral economics augment classical economic theory by incorporating psychological principles - often ascribed to cognitive limitations - to account for anomalous behavior (see, e.g., Kahneman \& Tversky, 2000; Camerer, Loewenstein, \& Rabin, 2004).

An alternative view is that people possess a repertoire of boundedly rational decision rules that they apply in specific circumstances (Gigerenzer \& Selten, 2001). Thus, boundedly rational rules can also produce appropriate responses. Specifically, Gigerenzer and his colleagues have demonstrated how what they call "fast and frugal rules" can rival the predictive ability of complex algorithms (Gigerenzer, Todd, \& the ABC Research Group, 1999). In their terms, bounded rationality can produce "ecologically rational" behavior, i.e., behavior that is appropriate in its "niche" but 
does not assume an underlying optimization model. What is unclear from this work, however, is where these niches are located in the regions of rationality.

Reviewing the empirical evidence, it is clear that there are occasions when boundedly rational agents fail to meet normative prescriptions as well as occasions when simple rules lead to surprisingly successful outcomes. The role of theory, therefore, is to specify the circumstances in which both kinds of results occur or, to use the metaphor of this paper, to map the regions of rationality.

Our goal is to illuminate this issue and our approach is theoretical. It involves specifying analytical models for simple processes that can be used for either multiattribute choice or multiple-cue prediction. Specifically, we derive probabilities that these models will correctly select the best of $m$ alternatives $(m \geq 2)$ based on $k$ attributes or cues $(k \geq 1)$. We also compare their effectiveness with optimizing and naïve benchmarks. The theoretical development enables the assessment of important environmental factors such as differential cue validities, inter-correlations of attributes, whether attributes/cues are measured by continuous or binary variables, levels of error in data, and the interactions between these factors.

This paper is organized as follows. In section I, we briefly review relevant literature. Next, in section II we specify the models we examine. In section III, we consider models based on continuous variables and derive conditions for choosing the best of three alternatives using a single variable prior to generalizing the number of alternatives and the use of different models. In section IV, we derive analogous conditions for models based on binary attributes or cues. In section V, we test our theories on twenty simulated and four empirical datasets and find excellent fits between predictions and realizations. We also provide an overview by regressing the performance of the different models on factors characterizing environments. Our 
results emphasize that relative model performance is a complex function of several factors and that theoretical models are needed to understand this complexity, i.e., to map the regions of rationality. For example, we identify regions where different models do and do not exhibit similar performance. At the same time, our results are consistent with some general trends that have been demonstrated previously in simulations (e.g., effects of inter-correlation among predictor variables). We also identify new regions where "less is more" (i.e., predictions are improved if less information is used). Finally, in section VI we suggest further research and conclude by commenting on implications of our work for the concept of rationality in economics.

\section{Evidence on the predictive effectiveness of simple models}

Interest in the efficacy of simple models for decision making has existed for some time with, in particular, numerous empirical demonstrations of how models based on simple equal (or unit) weighting schemes predict as well as or more accurately than more complex algorithms such as multiple regression (see, e.g., Dawes \& Corrigan, 1974; Dawes, 1979). Gigerenzer and Goldstein (1996) have further shown how a simple, non-compensatory lexicographic model that uses binary cues ("take the best" or TTB) is surprisingly accurate in predicting the better of two alternatives across several empirical datasets and outperforms the compensatory, equal weighting (EW) model (Gigerenzer et al., 1999).

Other studies have used simulation. Payne, Bettman and Johnson (1993), for example, explored tradeoffs between effort and accuracy. Using continuous variables and a weighted additive model as the criterion, they demonstrated the effects on simple model performance of two important environmental variables, dispersion in 
the weighting of variables and the extent to which choices involved dominance. (See also Thorngate, 1980). Based on conceptual considerations, Shanteau and Thomas (2000) defined environments as "friendly" or "unfriendly" to different models and also demonstrated these effects through simulations.

More recently, Fasolo, McClelland, and Todd (in press) examined multiattribute choice in a simulation using continuous variables (involving 21 options characterized by six attributes). Their goal was to assess how well choices by models with differing numbers of attributes could match total utility and, in doing so, they varied levels of average inter-correlations among the attributes and types of weighting functions. Results showed important effects for both. With differential weighting, one attribute was sufficient to capture at least $90 \%$ of total utility. With positive intercorrelation among attributes, there was little difference between equal and differential weighting. With negative inter-correlation, however, equal weighting was sensitive to the number of attributes used (the more, the better).

Despite these empirical demonstrations involving simulated and real data, research to date has generally lacked theoretical models for understanding how characteristics of models interact with those of environments. Some work has, however, considered specific cases. Einhorn and Hogarth (1975), for example, provided a theoretical rationale for the effectiveness of equal weighting relative to multiple regression. Martignon and Hoffrage (1999; 2002) and Katsikopoulos and Martignon (2003) explored the conditions under which TTB or equal weighting should be preferred in binary choice. Hogarth and Karelaia (2004; in press) and Baucells, Carrasco, and Hogarth (2005) have examined why TTB and other simple models perform well with binary attributes in error-free environments. And, Hogarth 
and Karelaia (2005) provided a theoretical analysis for the special case of binary choice with continuous attributes.

\section{Models considered}

The eleven specific models examined here are listed in Table 1. We distinguish five classes of models and, where possible, have versions based on both continuous and binary attributes/cues. ${ }^{2}$ Suffixes are used to distinguish the same models when used on the two kinds of data (i.e., $-c$ for "continuous" and $-b$ for "binary"). The DEBA model (number 3) is a deterministic version of Tversky's (1972) elimination-by-aspects (EBA) model. For binary choice, this model is identical to the TTB model of Gigerenzer and Goldstein (1996). With binary variables, DEBA frequently involves decisions based on a single attribute. In the continuous case this is best matched by the single variable model ( $\mathrm{SV}$, number 1) which is equivalent to the lexicographic model investigated by Payne et al. (1993). The presence of hybrids involving different models is represented by EW/DEBA and $\mathrm{EW} / \mathrm{SVb}$ (see also Hogarth \& Karelaia, in press). To provide lower benchmarks, we include two models that simply exploit dominance, Domran (DR), numbers 8 and 9. Finally, as a "normative" benchmark to calibrate our boundedly rational models we included multiple regression (models 10 and 11).

Insert Table 1 about here

It is important to emphasize that the models differ in the demands they make on cognitive resources, specifically on prior knowledge and the amount of

\footnotetext{
${ }^{2}$ In our simulations and empirical work, we generate binary variables by median splits of the continuous variables and in this manner make direct comparisons between results based on binary and continuous variables.
} 
information to be processed. We therefore indicate, on the right of Table 1, differential requirements in terms of prior information, information to consult, calculations, and numbers of comparisons to be made (minimum to maximum). For example, Table 1 shows that the EW and DR models require no prior information other than the signs of the zero-order correlations between the cues and the criterion (this is a minimum requirement). On the other hand, the lexicographic, DEBA, and hybrid models need to know which cue(s) is(are) most important. Against this, the lexicographic and DEBA models require less information and no calculations. The cost of DR models lies mainly in the number of comparisons that have to be made.

In this paper, we concentrate on accuracy or the probabilities that models make appropriate choices/predictions. However, and as demonstrated by Payne et al. (1993), it is important to bear in mind that heuristic models differ in their information processing costs.

\section{Models with continuous variables}

Choosing the best using a single variable (SV). For expository reasons, we consider first the case of selecting the best of three alternatives using a single variable (SV). Specifically, imagine choosing from a distribution characterized by two correlated random variables, one of which is a criterion, $Y$, and the other an attribute, $X$. Furthermore, assume that alternative $\mathrm{A}$ is preferred over alternatives $\mathrm{B}$ and $\mathrm{C}$ if $y_{a}>y_{b}$ and $y_{a}>y_{c}{ }^{3}$ Now, imagine that the only information about A, B, and C are the values that they exhibit on the attribute, $X$. Denote these specific values by $x_{a}, x_{b}$, and $x_{c}$, respectively. Without loss of generality, assume that $x_{a}>x_{b}$ and $x_{a}>x_{c}$ and that the decision rule is to choose the alternative with the largest value of $X$, i.e., in

\footnotetext{
${ }^{3}$ We denote random variables by upper case letters, e.g., $Y$ and $X$, and specific values or realizations by lower case letters, e.g., $y$ and $x$. As an exception, we use lower case Greek letters to denote random error variables, e.g., $\varepsilon$.
} 
this case A. The probability that $A$ is in fact the correct choice can therefore be characterized by the joint probability that $Y_{a}>Y_{b}$ given that $x_{a}>x_{b}$ and $Y_{a}>Y_{c}$ conditioned on $x_{a}>x_{c}$, in other words, $P\left\{\left(Y_{a}>Y_{b} \mid X_{a}=x_{a}>X_{b}=x_{b}\right) \cap\left(Y_{a}>Y_{c} \mid X_{a}=x_{a}>X_{c}=x_{c}\right)\right\}$.

To determine this probability, assume that $Y$ and $X$ are both standardized normal variables, i.e., both are $N(0,1)$. Moreover, the two variables are positively correlated (if they are negatively correlated, simply multiply one by -1 ). Denote the correlation by the parameter $\rho_{y x},\left(\rho_{y x}>0\right)$. Given these facts, it is possible to represent $Y_{a}, Y_{b}$, and $Y_{c}$ by the equations:

$$
\begin{aligned}
Y_{a} & =\rho_{y x} X_{a}+\varepsilon_{a} \\
Y_{b} & =\rho_{y x} X_{b}+\varepsilon_{b} \\
\text { and } \quad Y_{c} & =\rho_{y x} X_{c}+\varepsilon_{c}
\end{aligned}
$$

where $\varepsilon_{a}, \varepsilon_{b}$ and, $\varepsilon_{c}$ are normally distributed error terms, each with mean of 0 and variance of $\left(1-\rho_{y x}^{2}\right)$, independent of each other and of $X_{a}, X_{b}$, and $X_{c}$.

Using equations (1), (2), and (3) the differences between $Y_{a}$ and $Y_{b}$, on the one hand, and $Y_{a}$ and $Y_{c}$, on the other, can be written as

$$
Y_{a}-Y_{b}=\rho_{y x}\left(X_{a}-X_{b}\right)+\left(\varepsilon_{a}-\varepsilon_{b}\right)
$$

and

$$
Y_{a}-Y_{c}=\rho_{y x}\left(X_{a}-X_{c}\right)+\left(\varepsilon_{a}-\varepsilon_{c}\right)
$$

Thus, $Y_{a}>Y_{b}$ and $Y_{a}>Y_{c}$ if

$$
\rho_{y x}\left(X_{a}-X_{b}\right)>\varepsilon_{b}-\varepsilon_{a}
$$

and

$$
\rho_{y x}\left(X_{a}-X_{c}\right)>\varepsilon_{c}-\varepsilon_{a}
$$




$$
P\left\{\left(Y_{a}>Y_{b} \mid X_{a}=x_{a}>X_{b}=x_{b}\right) \cap\left(Y_{a}>Y_{c} \mid X_{a}=x_{a}>X_{c}=x_{c}\right)\right\} \quad \text { can now be }
$$

reframed as the probability that both the right hand side of (6) is smaller than $\rho_{y x}\left(X_{a}-X_{b}\right)$ and the right hand side of (7) is smaller than $\rho_{y x}\left(X_{a}-X_{c}\right)$. As can be seen, these latter terms are the products of $\rho_{y x}$, the correlation between $Y$ and $X$, and the differences between $X_{a}$ and $X_{b}$, and $X_{a}$ and $X_{c}$. In other words, the larger the correlation between $Y$ and $X$, and the larger the differences between $X_{a}$ and $X_{b}$, and $X_{a}$ and $X_{c}$, the greater $P\left\{\left(Y_{a}>Y_{b} \mid X_{a}=x_{a}>X_{b}=x_{b}\right) \cap\left(Y_{a}>Y_{c} \mid X_{a}=x_{a}>X_{c}=x_{c}\right)\right\}=$

$$
P\left\{\left(\varepsilon_{b}-\varepsilon_{a}<\rho_{y x}\left(x_{a}-x_{b}\right)\right) \cap\left(\varepsilon_{c}-\varepsilon_{a}<\rho_{y x}\left(x_{a}-x_{c}\right)\right)\right\}
$$

To determine this probability, we make use of the facts that the differences between the error terms, $\left(\varepsilon_{b}-\varepsilon_{a}\right)$ and $\left(\varepsilon_{c}-\varepsilon_{a}\right)$, are both normally distributed with means of 0 and variances of $2\left(1-\rho_{y x}^{2}\right)$. Standardizing $\left(\varepsilon_{b}-\varepsilon_{a}\right)$ and $\left(\varepsilon_{c}-\varepsilon_{a}\right)$, we can re-express equation (8) as

$$
\begin{aligned}
& P\left\{\left(\varepsilon_{b}-\varepsilon_{a}<\rho_{y x}\left(x_{a}-x_{b}\right)\right) \cap\left(\varepsilon_{c}-\varepsilon_{a}<\rho_{y x}\left(x_{a}-x_{c}\right)\right)\right\}= \\
& P\left\{\left(z_{1}<\frac{\rho_{y x}\left(x_{a}-x_{b}\right)}{\sqrt{2\left(1-\rho_{y x}^{2}\right)}}\right) \cap\left(z_{2}<\frac{\rho_{y x}\left(x_{a}-x_{c}\right)}{\sqrt{2\left(1-\rho_{y x}{ }^{2}\right)}}\right)\right\}
\end{aligned}
$$

where $z_{1}$ and $z_{2}$ are standardized normal variables with means of 0 and variances of 1 . Moreover, $z_{1}$ and $z_{2}$ jointly follow a bivariate normal distribution. Therefore, the target probability (9) can be written as

$$
\int_{-\infty}^{l_{a b} l_{a c}} \frac{1}{2 \pi \sigma_{z_{1}} \sigma_{z_{2}} \sqrt{1-\rho^{2}}} e^{-\frac{z}{2\left(1-\rho^{2}\right)}} d z_{1} d z_{2}
$$

where $\quad \mathrm{z}=\frac{z_{1}^{2}}{\sigma_{z_{1}}^{2}}-\frac{2 \rho z_{1} z_{2}}{\sigma_{z_{1}} \sigma_{z_{2}}}+\frac{z_{2}^{2}}{\sigma_{z_{2}}^{2}} ; \quad l_{a b}=\frac{\rho_{y x}\left(x_{a}-x_{b}\right)}{\sqrt{2\left(1-\rho_{y x}{ }^{2}\right)}} ; \quad l_{a c}=\frac{\rho_{y x}\left(x_{a}-x_{c}\right)}{\sqrt{2\left(1-\rho_{y x}{ }^{2}\right)}} ;$ $\sigma_{z_{1}}=\sigma_{z_{2}}=1$; and $\rho=\sigma_{z_{1}, z_{2}}$.

In Appendix A, we show that $\rho=1 / 2$. Thus, we can write 


$$
\begin{gathered}
P\left\{\left(Y_{a}>Y_{b} \mid X_{a}=x_{a}>X_{b}=x_{b}\right) \cap\left(Y_{a}>Y_{c} \mid X_{a}=x_{a}>X_{c}=x_{c}\right)\right\}= \\
\int_{-\infty-\infty}^{l_{a b} l_{a c}} \frac{1}{\pi \sqrt{3}} e^{-\frac{2}{3}\left(z_{1}^{2}-z_{1} z_{2}+z_{2}^{2}\right)} d z_{1} d z_{2}
\end{gathered}
$$

Figure 1 illustrates the probabilities of SV correctly choosing the best of three alternatives for different values of $\left(X_{a}-X_{b}\right)$ and $\left(X_{a}-X_{c}\right)$. In the panel on the left, (a), $\left(x_{a}-x_{c}\right)$ is held constant at a low value of 0.3 ; on the right, (b), it is held constant at a high value, 2.0. The lines in the figures reflect the effects of combining these fixed levels with different values of $\left(x_{a}-x_{b}\right)$, from 0.1 to 3.0. As can be observed, if one of the two differences, $\left(x_{a}-x_{b}\right)$ or $\left(x_{a}-x_{c}\right)$, is small, the probability of a correct choice varies between 0.4 and 0.5 . However, as both grow larger, so do the corresponding probabilities.

Insert Figure 1 about here

To generalize the above, assume that there are $m(m>3)$ alternatives from which to choose and that each has a specific $X$ value, $x_{l}, l=1, \ldots, m$. Without loss of generality, assume that $x_{1}$ has the largest value and we wish to know the probability that the corresponding alternative has the largest value on the criterion. Generalizing from the above, this probability can be calculated using properties of the multivariate normal distribution which, in this case, can be written,

$$
\begin{aligned}
& \int_{-\infty}^{d_{1}^{*}} \ldots \int_{-\infty}^{d_{m-1}^{*}} \varphi\left(z \mid \mu_{z}, V_{z}\right) d z_{1} \ldots . d z_{m-1}= \\
& \int_{-\infty}^{d_{1}^{*}} \ldots \int_{-\infty}^{d_{m-1}^{*}} \frac{\left|V_{z}\right|^{1 / 2}}{(2 \pi)^{(m-1) / 2}} e^{-\frac{1}{2} z^{\prime} V_{z}^{-1} z} d z_{1} \ldots . d z_{m-1}
\end{aligned}
$$


where $d_{i}^{*}=\frac{\rho_{y x} d_{i}}{\sqrt{2\left(1-\rho_{y x}^{2}\right)}}$ for $i=\overline{1, m-1}$, the elements of $z^{\prime}=\left(z_{1}, z_{2}, \ldots, z_{m-1}\right)$ are jointly distributed normal variables, with means of zero and variances of one, and $V_{z}^{-1}$ is the inverse of the $(m-1) \times(m-1)$ variance-covariance matrix where each diagonal element is equal to 1 and all off-diagonal elements equal $1 / 2$ (see Appendix A). In Appendix B we derive the analytical expression for the probability of selecting the optimal choice among four alternatives by using just one variable. For binary choice, that is, when $m=2$, analogous derivations lead to similar expressions to those shown above (see Hogarth \& Karelaia, 2005).

Overall probabilities. The probabilities given above are those associated with particular observations, i.e., that $\mathrm{A}$ is larger than $\mathrm{B}$ and $\mathrm{C}$ given that a specific value, $x_{a}$, exceeds specific values $x_{b}$ and $x_{c}$. However, it is also instructive to consider the overall expected accuracy of SV, i.e., the overall probability that SV makes the correct choice when sampling at random from the population of alternatives.

Successful predictions by SV can occur in three ways: predicting A when $x_{a}$ is bigger than $x_{b}$ and $x_{c}$; predicting $\mathrm{B}$ when $x_{b}$ is bigger than $x_{a}$ and $x_{c}$; and predicting $\mathrm{C}$ when $x_{c}$ is bigger than $x_{a}$ and $x_{b}$. The probabilities of these events are,

$$
\begin{aligned}
& P\left\{\left(\left(X_{a}>X_{b}\right) \cap\left(X_{a}>X_{c}\right)\right) \cap\left(\left(Y_{a}>Y_{b}\right) \cap\left(Y_{a}>Y_{c}\right)\right)\right\}, \\
& P\left\{\left(\left(X_{b}>X_{a}\right) \cap\left(X_{b}>X_{c}\right)\right) \cap\left(\left(Y_{b}>Y_{a}\right) \cap\left(Y_{b}>Y_{c}\right)\right)\right\}, \text { and } \\
& P\left\{\left(\left(X_{c}>X_{a}\right) \cap\left(X_{c}>X_{b}\right)\right) \cap\left(\left(Y_{c}>Y_{a}\right) \cap\left(Y_{c}>Y_{b}\right)\right)\right\}
\end{aligned}
$$

respectively, and the overall probability is the sum of the three terms. However, since each of the terms is equal to the others, the sum can be re-expressed as $3 P\left\{\left(\left(X_{a}>X_{b}\right) \cap\left(X_{a}>X_{c}\right)\right) \cap\left(\left(Y_{a}>Y_{b}\right) \cap\left(Y_{a}>Y_{c}\right)\right)\right\}$. 
To derive analytically the overall probability of correct choice by SV when sampling at random from the underlying population of alternatives, the latter expression should be integrated across all possible values that can be taken by $D_{1}=X_{a}-X_{b}>0$, and $D_{2}=X_{a}-X_{c}>0$. That is

$$
3 \int_{0}^{\infty} \int_{0}^{\infty} \varphi\left(d \mid \mu_{d}, V_{d}\right)\left[\int_{-\infty-\infty}^{d_{1}^{*} d_{2}^{*}} \varphi\left(z \mid \mu_{z}, V_{z}\right) d z_{1} d z_{2}\right] d d_{1} d d_{2}
$$

where $z^{\prime}=\left(z_{1}, z_{2}\right), V_{z}=\left(\begin{array}{cc}1 & 1 / 2 \\ 1 / 2 & 1\end{array}\right), d^{\prime}=\left(d_{1}, d_{2}\right), V_{d}=\left(\begin{array}{ll}2 & 1 \\ 1 & 2\end{array}\right), d_{1}^{*}=\frac{\rho_{y x} d_{1}}{\sqrt{2\left(1-\rho_{y x}^{2}\right)}}$, and $d_{2}^{*}=\frac{\rho_{y x} d_{2}}{\sqrt{2\left(1-\rho_{y x}^{2}\right)}} \cdot$ (In Table 3, discussed below, we generalize these formulas for choosing one of $m$ alternatives.)

Equal weighting (EW) and multiple regression (MR). What are the predictive accuracies of models that make use of several, $k$, cues or variables, $k>1$ ? We consider two models that have often been used in the literature. One is equal weighting (EW - see Dawes \& Corrigan, 1974; Einhorn \& Hogarth, 1975). The other is multiple regression (MR). To analyze these models, assume that the criterion variable, $Y$, can be expressed as a function

$$
Y=f\left(X_{1}, X_{2}, \ldots, X_{k}\right)
$$

where the $k$ predictor variables are multivariate normal, each with mean of 0 and standard deviation of 1 . For EW, the predicted $Y$ value associated with any vector of observed $x$ 's is equal to $\frac{1}{k} \sum_{j=1}^{k} x_{j}$ or $\bar{x}$. Similarly, the analogous prediction in MR is given by $\sum_{j=1}^{k} b_{j} x_{j}$ or $\hat{y}$ where the $b_{j}$ 's are estimated regression coefficients. In using 
these models, therefore, the decision rules are to choose according to the largest $\bar{x}$ for EW and the largest $\hat{y}$ value for MR.

How well are EW and MR likely to make the correct choice? Following the same rationale as the single variable (SV) case, we show in Table 2 the formulas used in deriving the analogous probabilities for EW and MR (as well as SV) when choosing the best of three alternatives using the properties of the bivariate normal distribution. These are the initial equations (corresponding to equations 1,2, and 3), the accuracy conditions (corresponding to equations 6 and 7), the relevant error variances, and finally the upper limits of integration, i.e. $l_{a b}$ and $l_{a c}$, used to calculate probabilities when applying equation (11).

Insert Tables $2 \& 3$ about here

Similarly, when calculating the probabilities of choosing correctly between four or more alternatives for $\mathrm{EW}$ and $\mathrm{MR}$, we can apply the multivariate normal distribution in analogous fashion to that of SV (cf. equation 12 above and Appendix B).

In Table 3, we present the formulas for the overall expected accuracy of EW and $\mathrm{MR}$ in a given environment or population, analogous to those for SV, i.e. to equation (13), for choosing one of $m$ alternatives. In particular, we present the elements that are specific for different models, such as the variance-covariance matrix, $V_{d}$, and the upper integration limits of integration, $d_{i}^{*}$. 


\section{Models with binary variables}

To discuss expected predictive performance of models based on binary variables, we first assume that the dependent variable, $Y$, can be thought of as being generated by a linear model of the form

$$
Y=\sum_{j=1}^{k} \gamma_{j} W_{j}+\varsigma
$$

where $W_{j}=0,1$ are the binary variables $(j=1, \ldots, k)$, the $\gamma_{j}$ are weighting parameters and $\varsigma$ is a normally distributed error term (see also below).

To derive theoretical predictions for models using binary variables, we adopt a similar approach to that used with continuous variables. We therefore focus on issues that differ between the continuous and binary cases.

Choosing the best using a single binary variable (SVb). Assuming that $w_{a}>w_{b}$ and $w_{a}>w_{c}$, the probability that $\mathrm{SVb}$ chooses correctly between three alternatives, $\mathrm{A}$, $\mathrm{B}$, and $\mathrm{C}$ is $P\left\{\left(Y_{a}>Y_{b} \mid W_{a}=w_{a}>W_{b}=w_{b}\right) \cap\left(Y_{a}>Y_{c} \mid W_{a}=w_{a}>W_{c}=w_{c}\right)\right\}$.

To determine this probability, recall that $Y$ is a standardized normal variable $N(0,1)$. The binary variable, $W$, however, only takes values of 0 and 1 and thus has a mean of 0.5 and standard deviation, $\sigma_{w}$, of $0.5{ }^{4}$ Denoting the correlation between $Y$ and $W$ by $\rho_{y w},\left(\rho_{y w}>0\right)$, we can express $Y$ by

$$
Y=\frac{\rho_{y w}}{\sigma_{w}} W+\varsigma
$$

or, simply, $\quad Y=2 \rho_{y w} W+\varsigma$

where $\varsigma$ is a normally distributed error term $N\left(-\rho_{y w}, 1-\rho_{y w}^{2}\right) .^{5}$

\footnotetext{
${ }^{4}$ Recall that binary variables are created by median splits of continuous variables.

${ }^{5} E(\varsigma)=-\rho_{y w}$, since $E(Y)=0=2 \rho_{y w} E(W)+E(\varsigma)=\rho_{y w}+E(\varsigma)$. Note, however, that the mean of the distribution of $\left(\varsigma_{b}-\varsigma_{a}\right)$ is 0 , i.e., $E\left(\varsigma_{b}-\varsigma_{a}\right)=-\rho_{y w}+\rho_{y w}$.
} 
Proceeding in similar fashion to the continuous case, we obtain the expression for the probability of SVb predicting correctly:

$$
\int_{-\infty-\infty}^{h_{a b} h_{a c}} \frac{1}{\pi \sqrt{3}} e^{-\frac{2}{3}\left(z_{1}^{2}-z_{1} z_{2}+z_{2}^{2}\right)} d z_{1} d z_{2}
$$

where $h_{a b}=\frac{2 \rho_{y w}\left(w_{a}-w_{b}\right)}{\sqrt{2\left(1-\rho_{y w}^{2}\right)}} ; \quad h_{a c}=\frac{2 \rho_{y w}\left(w_{a}-w_{c}\right)}{\sqrt{2\left(1-\rho_{y w}^{2}\right)}}$.

Since both $\left(w_{a}-w_{b}\right)$ and $\left(w_{a}-w_{c}\right)$ are equal to one, the two upper integration limits are the same: $h_{a b}=h_{a c}=\frac{2 \rho_{y w}}{\sqrt{2\left(1-\rho_{y w}^{2}\right)}}$.

As can be seen, the only difference between the theoretical expressions for the continuous and binary cases lies in the formulas for the upper limits of integration. Therefore, generalizing the above for choices among $m(m>3)$ alternatives is analogous to that for the continuous case.

Following the same rationale, we can derive the formulas for the probabilities for $\mathrm{EWb}$ and $\mathrm{MRb}$ when choosing the best of three alternatives using binary variables. In Table 4, we present the initial equations for these models (corresponding to equation 16), and the upper limits of integration, i.e. $h_{a b}$ and $h_{a c}$, used to calculate probabilities when applying equation (17).

Insert Table 4 about here

Choosing the best using DEBA with binary cues. Recall that this multi-stage model works in the following way: At the first stage, alternatives with values of 0 for the most important cue are eliminated unless all alternatives exhibit 0 . If only one alternative has a value of 1 , it is selected and the process terminates. If, however, more than one alternative remains, the same procedure takes place with the remaining 
alternatives except that the second most important cue is used. The process continues in the same manner through subsequent stages, if necessary. It stops when either only one alternative remains (i.e., the chosen alternative) or, if there is more than one alternative but no more cues, choice is determined at random among the remaining alternatives.

The probability that a given alternative was chosen correctly by DEBA is the probability that the sequence of decisions (or eliminations) made by the model at each stage is correct. Thus, since at each stage of the model decisions are made conditional on the preceding stages, the key parameters in estimating these probabilities are the partial correlations between $Y$ and $W_{j}, j=1, \ldots, k$ (i.e., controlling for previous stages). For the first stage, this is $\rho_{y w_{1}}$, for the second $\rho_{y w_{2} \cdot w_{1}}$, for the third, $\rho_{y w_{3}, w_{1} w_{2}}$, and so on. ${ }^{6}$

For example, assume that there are three alternatives $\mathrm{A}, \mathrm{B}$, and $\mathrm{C}$ and that $\mathrm{A}$ has been chosen by a process whereby $\mathrm{C}$ was eliminated at the first stage and $\mathrm{B}$ at the third stage. Starting backwards, consider the decisions the model makes at each stage. That is, the probability that DEBA correctly selected A over $\mathrm{B}$ at the third stage, controlling for the elimination of $\mathrm{C}$ at the first stage, is $P\left\{\left(Y_{a}>Y_{b} \mid W_{a 3}=w_{a 3}>W_{b 3}=w_{b 3}\right) \cap\left(Y_{b}>Y_{c} \mid W_{b 1}=w_{b 1}>W_{c 1}=w_{c 1}\right)\right\}$. This probability can be calculated by making use of the appropriate partial correlations - in this case, $\rho_{y w_{1}}$ and $\rho_{y w_{3}, w_{1} w_{2}}$ - and adapting the single variable equations (e.g., the general equation $10^{7}$ ). At the second stage, the model makes no decision. At the first stage, it eliminates $\mathrm{C}$ so we need to calculate additionally the probability that A could

${ }^{6}$ For example, $\rho_{y w_{2} . w_{1}}=\frac{\rho_{y w_{2}}-\rho_{y w_{1}} \rho_{w_{1} w_{2}}}{\sqrt{\left(1-\rho_{y w_{1}}^{2}\right)\left(1-\rho_{w_{1} w_{2}}^{2}\right)}}$.

${ }^{7}$ The terms that need to be adapted in the expression (10) are the upper limits or integration and $\sigma_{z_{1}, z_{2}}$. 
have been correctly selected only with information available at this stage: $P\left\{\left(Y_{a}>Y_{c} \mid W_{a 1}=w_{a 1}>W_{c 1}=w_{c 1}\right) \cap\left(Y_{c}>Y_{b} \mid W_{b 1}=w_{b 1}>W_{c 1}=w_{c 1}\right)\right\}$. This can be also found through an adapted expression (10), using $\rho_{y w_{1}}$. Importantly, the events represented by the probability expressions for the first and third stages are disjunctive. Therefore, the probability that DEBA makes the correct decision in this case is equal to the sum of the two expressions.

Consider another example involving three alternatives A, B, and C. Assume that DEBA eliminates $\mathrm{C}$ at the first stage and at the third stage picks either A or B at random (this will happen if $\mathrm{A}$ and $\mathrm{B}$ are identical). Thus, the 0.5 probability that DEBA makes the correct decision at the third stage should be "discounted" by the probability that $\mathrm{C}$, eliminated at the first stage, is not better than $\mathrm{A}$ and $\mathrm{B}$. That is $0.5\left(1-P\left\{\left(Y_{c}>Y_{a} \mid W_{c 1}=w_{c 1}>W_{a 1}=w_{a 1}\right) \cap\left(Y_{c}>Y_{b} \mid W_{c 1}=w_{c 1}>W_{b 1}=w_{b 1}\right)\right\}\right)$.

More generally, the probability of DEBA making the correct choice has to be calculated on a case-by-case basis taking into account, at each stage, the probability that the selected alternative should be chosen over the alternative(s) eliminated at that stage using the partial correlation of the cue appropriate to the stage. Moreover, the probability for each case includes the probabilities of successful decisions at each stage. If at the final stage, there are two or more alternatives, the appropriate random probability is adjusted by the probability that correct decisions were taken at previous stages (see, e.g., the example above). ${ }^{8}$

Choosing the best using the EW-SV model with binary cues (EW-SVb). The first stage of this model uses EWb. If a single alternative is chosen, the probability of it being correct is found by applying the formula for $\mathrm{EWb}$. If two or more alternatives

\footnotetext{
${ }^{8}$ In this section, we have only indicated the general strategy for calculating relevant probabilities for DEBA. The details involve repeated applications of the same probability theory principles applied in many different situations (Karelaia \& Hogarth, in preparation).
} 
are tied, a second stage consists of selecting the alternative favored by the first cue. To calculate the probability that this is correct, one needs to calculate the joint probability that the selected alternative (a) is larger than the alternatives eliminated at the first stage, and (b) larger than the other alternatives considered at the second stage. (To calculate these probabilities, use is made of the appropriate analogs to equation 17). Any ties remaining after stage two are resolved at random with a corresponding adjustment being made to the probability calculations.

Choosing the best using the EW-DEBA model with binary cues (EW-DEBA). This model starts as EWb. If EWb chooses one alternative, the probability of correct choice of the model coincides with that for $\mathrm{EWb}$. If two or more alternatives are tied, the DEBA model is used to choose between the remaining alternatives and probabilities are calculated accordingly (see above).

\section{Empirical evidence}

Our equations provide exact theoretical probabilities for assessing performance of the different models in specified conditions, i.e., to map the contours of the regions of rationality. However, several factors affect absolute and relative performance levels of the models (e.g., cue validities, ${ }^{9}$ inter-correlation among variables, continuous vs. binary variables, error), and it is difficult to assess their importance simply by inspecting the formulas.

We therefore use both simulated and empirical data to illuminate model performance under different conditions. Real data have the advantage of testing the theory in specific, albeit limited environments. Simulated data, on the other hand,

\footnotetext{
${ }^{9}$ The cue validity for a particular predictor variable is defined by its correlation with the criterion.
} 
facilitate testing model predictions over a wide range of environments. We first consider the simulated data.

Simulation design and method. The simulation design used for choosing the best from two, three and four alternatives is presented in Table 5. Overall, we specified 20 different populations that are subdivided into four sets or cases $-\mathrm{A}, \mathrm{B}, \mathrm{C}$, and $\mathrm{D}$ - each of which contains five sub-cases (labeled 1, 2, 3, 4, and 5). Since, a priori, several factors might be thought important, we would have liked to vary these orthogonally. However, correlations between factors restrict implementing a fully systematic design. We therefore varied some factors at the level of the cases (A, B, C, and D) and others across sub-cases (i.e., within A, B, C, and D).

At the level of cases, A and B involved three cues or attributes whereas cases $\mathrm{C}$ and D involved five. Cases A and $\mathrm{C}$ had little or no inter-cue correlation; cases B and D had moderate to high intercorrelation.

Insert Table 5 about here

Across sub-cases (i.e., from 1 through 5 within each of A, B, C, and D), we varied: (1) the variability of cue validities (maximum - minimum); (2) the validity of the first (i.e., most important) cue; (3) average validity; and (4) the correlation between $y$ and $\bar{x}$. For all, values increase from the sub-cases 1 through 5 . As a consequence, the $\mathrm{R}^{2}$ on initial fit for MR also increases across sub-cases. This implies that the sub-cases 1 involve high levels of error whereas the sub-cases 5 are, in principle, quite predictable environments. Sub-cases 2, 3, and 4 fall between these extremes.

To conduct the simulation, we defined 20 sets of standardized multivariate normal distributions with the parameters specified in Table 5 and generated samples 
of size 40 from each of these populations. The observations in each sample were split at random on a 50/50 basis into fitting and prediction sub-samples and model parameters were estimated on the fitting sub-sample. Two, three or four alternatives (as appropriate) were then drawn at random from this sub-sample and, using the estimated model parameters, probabilities of correctly selecting the best of these specific alternatives were calculated. This was then compared to what actually happened, that is, on a "fitting" basis. Next, alternatives were drawn at random from the prediction sub-sample, relevant probabilities calculated using the parameters from the fitting sub-sample, and predictions compared to realizations. This exercise was repeated 5,000 times (for each of the choices involving two, three, and four alternatives).

The above describes the procedure used for continuous data. For models using binary data, we followed exactly the same procedures except that predictor variables only took values of 0 or 1 . Specifically, since we were sampling continuous normalized variables, we created binary variables by median splits (i.e., binary variables were set to 0 for negative values of continuous variables and 1 for nonnegative values). Thus, if one estimates the parameters of the 20 populations for the binary data, the estimates differ systematically from their continuous counterparts shown in Table 5 (they are smaller). However, we do not display the binary parameter estimates since the parameters in Table 5 represent the process that generated the data.

Simulation results. Tables 6,7 , and 8 present the results of the simulations for the choice of best of two, three, and four alternatives, respectively. Figure 2 presents some selected outcomes from the case involving best of three (Table 7). Results 
reported here are limited to predictions and realizations for the holdout samples (i.e., tests of cross-validation). ${ }^{10}$

To simplify reading the tables, note that realizations are underlined (e.g., $\underline{62}$ ), and that the largest realization for each population (i.e., per column) is presented in bold (e.g., $\underline{\mathbf{6 5}}$ ). In addition, when MRc is the largest, we also denote the second largest in bold. We further show mean realizations for each column and row in the tables. The column means thus represent the average realizations of all models within specific populations whereas the row means characterize average model performance across populations.

We first note that, with the exception of multiple regression (MR), the match between model predictions and realizations is quite close in all cases. MR makes large errors for cases $\mathrm{C}$ and $\mathrm{D}$ and particularly when the differences between maximum and minimum cue validities are smallest (sub-cases 1 and 2). Although we used adjusted $\mathrm{R}^{2}$ in making predictions, the adjustment was insufficient in these situations. ${ }^{11}$

Insert Tables 6, 7, 8 and Figure 2 about here

Qualitatively, the relative effectiveness of the models is quite similar whether one looks at the results for best of two, three, or four. What changes, of course, is the general level of performance which diminishes as the number of alternatives increases. This can be seen by comparing the columns of mean realizations of Tables 6,7 , and 8 , i.e., at the extreme right hand sides of the tables.

\footnotetext{
10 As might be expected by examining the success of the models on cross-validation, the fitting exercise produced almost perfect matches between samples and models.

${ }^{11}$ One can also argue with some justification that the ratio of observations to predictor variables is too small to use multiple regression (particularly for cases $\mathrm{C}$ and D). However, we are particularly interested in observing how well the different models work in environments where there is not much data.
} 
Within each table, an initial, overall impression is the lack of large differences between the performances of the different models. However, there are systematic effects.

First, consider whether predictor variables are binary or continuous. The use of binary as opposed to continuous variables implies a loss of information. As such, models based on continuous variables would be expected to predict better than their binary counterparts. Indeed, this is always the case in three direct comparisons: SVc vs. SVb, EWc vs. EWb, and MRc vs. MRb. Specifically, note that SVb and EWb are both handicapped relative to SVc and EWc in that they necessarily predict many ties that are resolved at random. Thus, so long as the knowledge in SVc and EWc implies better than random predictions, models based on continuous variables are favored.

On the other hand, the performance of DRb dominates DRc for cases A, C, and D with performance being quite similar for case B (for best of two, three, and four). It would appear that DRb exploits more cases of "apparent" dominance than DRc which (as a consequence) decides more choices at random. Thus, to the extent that the "additional" dominance cases detected by DRb relative to DRc have more than a random chance of being correct, DRb outpredicts DRc. Whereas the DR models represent naïve baseline strategies, we believe this finding is important because it demonstrates how a simple strategy can exploit the structure of the environment such that more information (in the form of continuous as opposed to binary variables) does not improve performance (a so-called "less is more" effect, Goldstein \& Gigerenzer, 2002; Hertwig \& Todd, 2003).

A second interesting set of comparisons is between SVc and DEBA. The former uses a single, continuous variable. The latter relies heavily on one binary variable but can also use others depending on circumstances. It is thus difficult to 
state which strategy actually uses more information. However, once again, characteristics of the environment determine which strategy is more successful. SVc dominates DEBA in case B as well as for much of cases A and D. On the other hand, DEBA dominates SVc in case C.

In Figure 2, we have chosen to illustrate the performance of five models across all the environments for the choice of best of three (i.e., data from Table 7). Two of these models, DRb and MRc are depicted because they represent, respectively, naïve and "sophisticated" benchmarks. The other models, SVc, DEBA, and EWc are quite different types of heuristics (see Table 1). Both SVc and DEBA require prior knowledge of what is important (DEBA more so than SVc). However, they use little information and neither involves any computation. (DEBA, it should be recalled, also operates on binary data.) EWc, on the other hand, does not require knowledge of differential importance of variables but does use all information available and needs some computational ability.

When environments contain much error (the sub-cases 1), the performances of all models are degraded such that differences are small. However, as error decreases (i.e., moving right toward the sub-cases 5), model performances vary by environmental conditions. In the absence of inter-cue correlation (cases A and C), there appear to be large differences in model performance. However, in the presence of such correlations (cases B and D), there are two distinct classes of models: SVc and MRc have similar performance levels and are superior to the others. We further note that $\mathrm{SVc}$ is most effective in case $\mathrm{B}$ and also does well as environmental predictability increases in cases A and D. DEBA is never the best model but performs quite adequately in case $\mathrm{C}$ where $\mathrm{EWc}$ has the best performance. Of the benchmark models, DRb generally lags behind the other models (as would be expected). Finally, 
although MRc is typically one of the better models, it does not dominate in all environments.

To highlight regions of rationality, Table 9 represents the data from Figure 2 in another manner (think of Table 9 as a map!). Specifically, the performances of SVc, DEBA, EWc, and DRb are compared to the "normative" benchmark of MRc (by deducting the performance of each of the former from the latter). Thus positive (negative) entries in Table 9 indicate the amount by which the performance of MRc exceeds (falls short of) those of other models. Three cases are indicated: in the shaded areas, MRc exceeds other models by at least 5; in the unshaded areas, the standard font (e.g., 4) indicates that the MRc advantage is small $(<5)$ but positive; and bold, underlined font (e.g., $\underline{\mathbf{2}}$ ) denotes that MRc has no advantage over the other model. The table depicts relative model performance by characteristics of regions, e.g., redundancy and noise (that is, inter-cue correlation and error, respectively).

Insert Table 9 about here

For example, recall from Table 5 that cases $\mathrm{A}$ and $\mathrm{C}$ involve low redundancy whereas this is not true of cases B and D. Also, for all cases, as one moves from subcases 1 through 5, environments involve less noise. With this in mind, one can attribute the smaller differences between models in sub-cases 1 and 2 as being mainly due to the effects of error. Interestingly, the relative success of SVc in cases B and D (compared to A and C) seems to be the effect of inter-correlation between the other attributes, i.e., redundancy. Table 9 is only presented as an illustration. The data from Tables 6,7 , and 8 can clearly be used to create maps that highlight different aspects of the decision making terrain. 
A further way of summarizing factors that affect the performance of the different models is to consider the regression of model performance on statistics that describe the characteristics of the 20 simulated environments (Table 5). In other words, consider regressions for each of our eleven models of the form

$$
P_{i}=Z \underline{\delta_{i}}+\tau_{i}
$$

where $P_{i}$ is the performance realization of model $i(i=1, \ldots, 11) ; Z$ is the $(20 \times 3) \times s$ matrix of independent variables (statistics characterizing the datasets where there are three choice situations, i.e., best of two, three, or four alternatives); $\underline{\delta_{i}}$ is the $s \mathrm{x} 1$ vector of regression coefficients; and $\tau_{i}$ is a normally distributed error term with constant variance, independent of $Z$.

To characterize the environments or datasets (the $Z$ matrices), we chose the following variables: variability of cue validities $(\max -\min )$, the validity of the most important cue $\left(r_{y x_{1}}\right)$, the validity of the average of the cues $\left(r_{y \bar{x}}\right)$, average intercorrelation of the cues, average validity of the cues, number of cues, and $\mathrm{R}^{2}$ for MRc and MRb. ${ }^{12}$ We also used dummy variables to model the effects of choosing between different numbers of alternatives. Dummyl captures the effect of choosing from three as opposed to two alternatives, and Dummy2 the additional effect of choosing from four alternatives. Results of the regression analyses are summarized in Table 10.

Insert Table 10 about here

\footnotetext{
12 We only used $\mathrm{R}^{2}$ as an independent variable for MRc and MRb because we thought it would be appropriate for these models. For the other models, however, it was deemed more illuminating to characterize performance by the other measures $\left(\mathrm{R}^{2}\right.$ and these other measures are correlated in different ways). It should also be noted that we used the same statistics (based on continuous variables) to characterize the environments for models using both continuous and binary variables on the grounds that the underlying environments were based on continuous variables.
} 
We used a step-wise procedure with entry (exit) thresholds for the variables of $<.05(>.10)$ for the probability of the F statistic. All coefficients for the models shown in Table 10 are statistically significant $(\mathrm{p}<.001)$ and all regressions exhibited excellent fits overall (see $\mathrm{R}^{2}$ and estimated standard errors at the foot of Table 10). The constant term is fairly high across all models and measures the level of performance that would be expected of the models in binary choice absent information about the environment (approximately 50, i.e., from 42 to 65). Dummy1 indicates how much such performance would fall when choosing between three alternatives (between 11 and 14), and Dummy2 shows the additional drop experienced when choosing among four alternatives (between 6 and 9).

For SVc and SVb, only one other variable is significant, the correlation between the single variable and the criterion. This makes intuitive sense as does the fact that the regression coefficient is larger with continuous as opposed to binary variables (50 vs. 29). The DEBA and EW models are all heavily influenced by the correlation between the criterion and $\bar{x}$. Recall, however, that this correlation is itself an increasing function of average cue validity and the number of cues but decreasing in the inter-correlation between cues (see the formula in footnote 1 to Table 2). Thus, ceteris paribus, increasing inter-correlation between the cues reduces the absolute performance levels of these models. DEBA differs from the EW models in that the correlation of the most valid cue is a significant predictor. This matches expectations in that DEBA relies heavily on the validity of the most important cue whereas EW weights all cues equally. (We also note that the SV models weight the most valid cue more heavily than DEBA.) As to the Domran models, the interpretation of the signs of all coefficients is not obvious. Finally, for MRc it comes as little surprise that $\mathrm{R}^{2}$ should be so important although this variable is less salient for MRb. 
A possible surprise is that variability in cue validities (maximum less minimum) was not a significant factor for most models. One might have thought, a priori, that such dispersion would have been important for DEBA (cf., Payne et al., 1993). However, this is not the case and is consistent with theoretical analyses of DEBA that show that its accuracy is relatively robust to different "weighting functions" (Hogarth \& Karelaia, in press; Baucells, Carrasco, \& Hogarth, 2005).

Finally, whereas the regression statistics paint an interesting picture of model performance in the particular environments observed, we caution against overgeneralization. We only observed restricted ranges of the environmental statistics (i.e., characteristics) and thus cannot comment on what might happen beyond these ranges. Our approach, however, does suggest a way to illuminate model $\mathrm{x}$ environment interactions.

To summarize, across all 20 environments that, inter alia, are subject to different levels of error, the relative performances of the different models were not seen to vary greatly when faced with the same tasks (e.g., choose best of three alternatives). However, there were systematic differences due to interactions between characteristics of models and environments. Thus, whereas the additional information contained in continuous as opposed to binary variables benefits some models, e.g., SV and EW, it can be detrimental to others, e.g., DR. Second, models varied in the extent to which they were affected by specific environmental characteristics. SV models, for example, depend heavily on the validity of the most valid cue whereas this only affects EW models through its impact on average cue validity. Interestingly, the validity of the average of the cues was seen to have more impact on the performance of DEBA than the validity of the most valid cue. Average inter-correlation of predictors or redundancy tends to reduce performance of all models (except SV). 
Overall, results do match some general trends noted in previous simulations (Payne et al., 1993; Fasolo et al., in press); however, patterns are not simple to describe. The value of our work, therefore, is that we now possess the means to make precise predictions for various simple, boundedly rational models in different environments. That is, given specified environments, we can predict a priori both levels of model performance and which models will be more or less effective.

An important environmental factor we did not vary was the impact of different distributions of specific kinds of alternatives. Instead, we simulated random drawings of alternatives given the population characteristics defined in Table 5. We did not, for example, skew the sampling process to include or exclude disproportionate numbers of, say, dominating or dominated alternatives (cf., Payne et al., 1993). As we have argued elsewhere (Hogarth \& Karelaia, 2004), the distribution of alternatives can have important effects on both the general level of performance achieved by models as well as relative performance (some distributions, for example, are relatively "friendly" or "unfriendly" to specific models, Shanteau \& Thomas, 2000). On the other hand, since our methodology can make specific predictions for each case encountered, it can easily handle the effects of sampling from different distributions of alternatives.

Empirical data. We used datasets from three different areas of activity. The first involved performance data of the 60 leading golfers in 2003 classified by the Professional Golf Association (PGA) in the USA. ${ }^{13}$ From these data $(\mathrm{N}=60)$, we examined two dependent variables: "all-round ranking" and "total earnings." The first is a measure based on eight performance statistics. For our models, we chose three predictor variables that account for $67 \%$ of the variance in the criterion. These were

\footnotetext{
13 These data were obtained from the webpage http://www.pgatour.com/stats/leaders/r/2003/120. They are performance statistics of golfers in the main PGA Tour for 2003.
} 
mean numbers, across rounds played, of birdies, total scores, and putts. Since the first variable was negatively related to the criterion, it was rescaled (multiplying by -1).

Eighty-two percent of the variance in the second golf variable, total earnings, could be explained by three variables, "number of top 10 finishes," "all-round ranking" (the previous dependent variable), and "number of consecutive cuts." Of these, all-round ranking was negatively correlated with the criterion and so rescaled (multiplying by -1 ).

The second dataset consisted of rankings of $\mathrm{PhD}$ economics programs in the USA on the basis of a 1993 study by the National Research Council $(\mathrm{N}=107) .{ }^{14}$ Three variables accounted for $80 \%$ of the variance in the rankings: number of $\mathrm{PhD}$ 's produced by programs for the academic years 1987-88 through 1991-1992, total number of program citations in the period 1988-1992 divided by number of program faculty, and percentage of faculty with research support.

The third dataset was taken from the UK consumer organization Which?'s assessments of digital cameras in $2004(\mathrm{~N}=49) .{ }^{15}$ Three variables were found to explain $72 \%$ of the variance in total test scores: image quality, picture downloading time, and focusing.

Table 11 summarizes statistical characteristics of these datasets (analogous to the experimental design of the simulations in Table 5). As can be seen, there is little to moderate variability in cue validities (compare Economics PhD programs with the other datasets); all datasets have at least one highly valid cue; average intercorrelation varies from moderate to low (Consumer reports: Digital cameras); and there are high correlations between the criteria and the means of the predictor variables.

\footnotetext{
${ }^{14}$ For more details, see the webpage http://www.phds.org

${ }^{15}$ For further details, see the webpage http://sub.which.net
} 
The testing procedure was similar to the simulation methodology. We divided each sample at random on a 50/50 basis into fitting and testing sub-samples. Model parameters were then estimated on the fitting sub-sample and these parameters used to calculate the probabilities that the models would correctly choose the best of two, three, and four alternatives that had also been randomly drawn from the same subsample. This was the fitting exercise. To test the models' predictive abilities, two, three, and four alternatives were drawn at random from the second or testing subsample and, using the parameters estimated from the data in the first or fitting subsample, model probabilities were calculated for the specific cases and subsequently compared to realizations. This exercise was repeated 5,000 times such that the data reported in Table 12 shows the aggregation of all these cases. Also similar to the simulation methodology, we created binary datasets by using median splits of the continuous variables.

Insert Tables 11, 12 and Figure 3 about here

Table 12 has been constructed in similar fashion to Tables 6, 7, and 8 except that we show results for choosing the best out of two, three, and four alternatives within columns for each dataset. Figure 3 also graphs results for select models for each dataset.

Once again, there is an excellent fit between predictions and realizations. ${ }^{16}$ The largest differences occur, as before, with MR. Overall, the models have fairly high levels of performance which, naturally, diminish as the number of alternatives increases (however, perhaps, not as much as might have been thought $a$ priori. See, in particular, Economics PhD programs). As with the simulated datasets, the SV, EW,

\footnotetext{
${ }^{16}$ For this reason, we do not present here the excellent fits achieved by the models on the fitting samples.
} 
and MR models with continuous variables outperform their binary counterparts (with one exception in 36 comparisons) but DRb dominates DRc. As to the SVc versus DEBA comparison, SVc outperforms DEBA by some margin on the Golf rankings and Golf earnings datasets but DEBA dominates SVc on the other two datasets. From a statistical viewpoint, these two datasets differ from the others in that the Economics PhD programs has low variability of cue validities and Consumer reports: Digital Cameras has low average cue inter-correlation.

Differences between models on the different datasets are highlighted in Figure 3 where we again show the performances of SVc, DEBA, EWc, DRb, and MRc. Although the datasets have some similarities (e.g., the validities of the first cue and the correlations between the criteria and the means of the predictor variables are almost the same), other differences (notably variability in cue validities and levels of cue inter-correlations) are sufficient to change the relative effectiveness of the models. SVc is very effective for the Golf rankings and Golf earnings data, EWc predicts the Economics PhD programs well and DEBA is best for the Consumer reports: Digital Cameras data. As to the two benchmark models, DRb generally has the lowest performance but, with the exception of the Consumer reports: Digital cameras dataset, is close to DEBA. For all datasets, MRc is always one of the better models but it does not dominate the other models. Finally, we emphasize once again the close fit between our model predictions and the empirical realizations demonstrated in Table 12. Taken as a whole, our theoretical models account for complex patterns of data. 


\section{Discussion}

We have mapped regions of rationality by studying a class of decisions that involve choosing the best of $m(m \geq 2)$ alternatives on the basis of $k(k \geq 1)$ cues or attributes. As such, this is a common task in inference and also has applications to preference (cf., Hogarth \& Karelaia, in press). We have shown - through theory, simulation, and empirical demonstration - that certain simple, boundedly rational models can have effective performance relative to more complex, sophisticated benchmarks and, indeed, when data are scarce can, on occasion, perform better than the latter. More importantly, our theoretical analysis predicted differential model performance in a wide range of environments. Thus, for example, for our empirical datasets we predicted - and later verified - that EWc would be the best of the simple models for Economics PhD programs but SVc the best for Golf rankings.

General trends concerning relative model performance have, of course, been known for some time (e.g., effects of inter-correlations between cues or attributes, cf., Einhorn \& Hogarth, 1975; Payne et al., 1993). However, the advantage of our approach is that we can specify a priori the combined effects of different environmental characteristics such as variability in cue validities, inter-correlations, level of error, and so on. Moreover, we observed that the effects of "tradeoffs" between such factors are complex and often defy simple description. The terrain that we have mapped has many dimensions.

One factor we did not consider was the effects of sampling alternatives from the underlying populations in biased or non-random ways. Clearly, results would be different if sampling excluded certain profiles of alternatives such as those likely to 
dominate others or be dominated. ${ }^{17}$ On the other hand, our theoretical method allows us to make case-by-case predictions such that - through suitable aggregation - we could make predictions for samples drawn in specific, non-random ways provided the same sampling procedures are used in both fitting and holdout samples. Showing the effects of such non-random sampling is thus a straightforward task that can be addressed in future research.

The present paper is also limited by the criterion used to measure model effectiveness, i.e., the emphasis on probability of correct choices. This might seem restrictive in that it assumes a "hit or miss" criterion with no consideration as to how "good" the other alternatives are. We accept this limitation. However, we point out that our theoretical models can be used to specify not just the probability that one alternative will be correctly selected but also the probabilities for all alternatives. For example, imagine choosing between three alternatives A, B, and C using the SV model and having observed $x_{a}>x_{b}>x_{c}$. Above, we calculated the probability $P\left\{\left(Y_{a}>Y_{b} \mid X_{a}=x_{a}>X_{b}=x_{b}\right) \cap\left(Y_{a}>Y_{c} \mid X_{a}=x_{a}>X_{c}=x_{c}\right)\right\}$. However, we could also have calculated the probability that $\mathrm{B}$ is the largest, that is $P\left\{\left(Y_{b}>Y_{a} \mid X_{a}=x_{a}>X_{b}=x_{b}\right) \cap\left(Y_{b}>Y_{c} \mid X_{b}=x_{b}>X_{c}=x_{c}\right)\right\}$ and so on. In other words, we can specify the probabilities associated with all possibilities. Given such distributions over possible outcomes, it is straightforward to consider the effects of different loss functions, a topic we also leave for further research.

Future work could also build on our theoretical approach to consider variations of the models we have examined here. For example, models might involve mixtures of categorical and continuous variables or the effects of different types of error. How,

\footnotetext{
${ }^{17}$ Note that we would not be able to apply our "overall formulas" (e.g., equation 13) to these populations because they assume unbiased, random sampling.
} 
for instance, would simple models perform when there are errors in the variables (perhaps due to measurement problems) or missing values? Clearly many further elaborations could be undertaken.

Our work has particular implications for the economics of information and the meaning of rationality when attention is a scarce resource. As stated by Simon (1978):

In a world in which information is relatively scarce, and where problems for decision are few and simple, information is almost always a positive good. In a world where attention is a major scarce resource, information may be an expensive luxury, for it may turn our attention from what is important to what is unimportant. We cannot afford to attend to information simply because it is there (Simon, 1978, p. 13).

By way of illustration, Simon described executives whose management information systems provide excessive, detailed information. Our work also identified regions where more information does not necessarily lead to better decisions and, if we assume that more complex models require more cognitive effort (or computational cost), there are many areas where there is no tradeoff between accuracy and effort. For example, in cases B and D illustrated in Figure 2, the simple SVc model is more accurate than the other models indicated across almost the whole range of conditions and, yet, it uses less information. On the other hand, EWc is generally best in case C where SVc lags behind the other models. However, EWc uses more information than both SVc and DEBA such that one can ask whether the additional predictive ability is worth its cost.

The models we examined might also be used in applied areas such as consumer research (cf., Bettman, Luce, \& Payne, 1998). That is, instead of assuming that consumers make tradeoffs across many attributes, simpler SV or EW models can be constructed after eliciting a few simple questions concerning, say, relative importance of attributes. For example, we have shown elsewhere that if people have loose preferences characterized by binary attributes, the outputs of DEBA are 
remarkably consistent with more complex, linear tradeoff models (Hogarth \& Karelaia, in press). However, it would be a mistake to assume that consumer preferences can always be modeled by one simple, boundedly rational model (e.g., EW). Indeed, our theoretical analysis provides the basis for deciding which models are suited to different environments.

Finally, in a world where attention is the scarce resource, we note that "rational behavior" consists of finding the appropriate match between a simple decision rule and the task with which it is confronted. In describing economic decision making, therefore, we do not need to assume unlimited computational capacity. However, by relaxing this assumption, the researcher incurs two costs. The first, analyzed in this paper, is to identify the task conditions under which specific heuristic rules are and are not effective, i.e., to develop maps of the regions of rationality. The second, that awaits further research, is to elucidate the conditions under which people do or do not acquire such knowledge. In other words, how do agents build maps of their decision making terrain? To be effective, "boundedly rational" agents do not need much computational ability to make choices in the mazes that define their environments. However, they do need task-specific knowledge or maps. 


\section{References}

Baucells, M., Carrasco, J. A., \& Hogarth, R. M. (2005). Cumulative dominance and heuristic performance in binary multiattribute choice. Barcelona: Working paper IESE, UPF, and UPC.

Bettman, J. R., Luce, M. F., \& Payne, J. W. (1998). Constructive consumer choice processes. Journal of Consumer Research, 25, 187-217.

Camerer, C. (1998). Bounded rationality in individual decision making. Experimental Economics, 1, 163-183.

Camerer, C. F., Loewenstein, G., \& Rabin, M. (2004). (Eds.), Advances in Behavioral Economics. New York and Princeton: Russell Sage Foundation and Princeton University Press.

Conlisk, J. (1996). Why bounded rationality? Journal of Economic Literature, 34 (2), $669-700$.

Dawes, R. M., (1979). The robust beauty of improper linear models. American Psychologist, $\underline{34}$, 571-582.

Dawes, R. M., \& Corrigan, B. (1974). Linear models in decision making. Psychological Bulletin, 81, 95-106.

Einhorn, H. J., \& Hogarth, R. M. (1975). Unit weighting schemes for decision making. Organizational Behavior and Human Performance, $\underline{13}$, 171-192.

Fasolo, B., McClelland, G. H., \& Todd, P. M. (in press). Escaping the tyranny of choice: When fewer attributes make choice easier. Marketing Theory.

Gigerenzer, G., \& Goldstein, D. G. (1996). Reasoning the fast and frugal way: Models of bounded rationality. Psychological Review, 103, 650-669. 
Gigerenzer, G., \& Selten, R. (2001). (Eds.) Bounded rationality: The adaptive toolbox. Cambridge, MA: The MIT Press.

Gigerenzer, G., Todd, P. M., and the ABC Research Group. (1999). Simple heuristics that make us smart. New York: Oxford University Press.

Goldstein, D. G., \& Gigerenzer, G. (2002). Models of ecological rationality: The recognition heuristic. Psychological Review, 109 (1), 75-90.

Hertwig, R., \& Todd, P. M. (2003). More is not always better: The benefits of cognitive limits. In D. J. Hardman \& L. Macchi (eds.), Thinking: Psychological perspectives on reasoning, judgment and decision making. Chichester, England: Wiley.

Hogarth, R. M., \& Karelaia, N. (2004). "Take-the-Best” and other simple strategies: Why and when they work "well" in binary choice. Barcelona: Universitat Pompeu Fabra, DEE working paper.

Hogarth, R. M., \& Karelaia, N. (2005). Ignoring information in binary choice with continuous variables: When is less "more"? Journal of Mathematical Psychology, $\underline{49}, 115-124$.

Hogarth, R. M., \& Karelaia, N. (in press). Simple models for multi-attribute choice with many alternatives: When it does and does not pay to face tradeoffs with binary attributes. Management Science.

Kahneman, D. (2003). Maps of bounded rationality: Psychology for behavioral economics. American Economic Review, 93(5), 1449-1475.

Kahneman, D., Slovic, P., \& Tversky, A. (1982). (Eds.) Judgment under uncertainty: Heuristics and biases. New York: Cambridge University Press.

Kahneman, D., \& Tversky, A. (2000). (Eds.) Choices, values, and frames. New York: Cambridge University Press \& Russell Sage Foundation. 
Karelaia, N., \& Hogarth, R. M. (in preparation). On predicting performance of DEBA models in the presence of error. Barcelona: Universitat Pompeu Fabra

Katsikopoulos, K., \& Martignon, L. (2003). Paired comparisons: Ordering or adding attributes? Working paper, Max-Planck Institute for Human Development, Berlin.

Martignon, L., \& Hoffrage, U. (1999). Why does one-reason decision making work? A case study in ecological rationality. In G Gigerenzer, P. M. Todd and the ABC Research Group. Simple heuristics that make us smart (pp. 119-140). New York: Oxford University Press.

Martignon, L., \& Hoffrage, U. (2002). Fast, frugal, and fit: Simple heuristics for paired comparison. Theory and Decision, $\underline{52}, 29-71$.

Payne, J. W., Bettman, J. R., \& Johnson, E. J. (1993). The adaptive decision maker. New York: Cambridge University Press.

Shanteau, J., \& Thomas, R. P. (2000). Fast and frugal heuristics: What about unfriendly environments? Behavioral and Brain Sciences, 23, 762-763.

Simon, H. A. (1956). Rational choice and the structure of environments. Psychological Review, 3, 129-138.

Simon, H. A. (1978). Rationality as process and product of thought. American Economic Review, $\underline{68}(2), 1-16$

Simon, H. A. (1991). Models of my life. New York: Basic Books.

Starmer, C. (2000). Developments in non-expected utility theory: The hunt for a descriptive theory of choice under risk. Journal of Economic Literature, $\underline{38}$ (2), $332-382$.

Thorngate, W. (1980). Efficient decision heuristics. Behavioral Science, 25, 219-225.

Tversky, A. (1972). Elimination by aspects: A theory of choice. Psychological Review, 79, 281-299. 


\section{Table 1 -- Models tested}

$\underline{\text { Single Variable (SV) models }}$

1 Lexicographic Choice depends solely on cue with the greatest validity (see, e.g., Payne et -- SVc

al., 1993).

2 Lexicographic Model 1 above but based on binary variables.

$--\mathrm{SVb}$

3 DEBA

Deterministic version of Tversky's (1972) elimination-by-aspects model

(EBA). For binary choice, this is the same as the take-the-best model of

Gigerenzer and Goldstein (1996).

Equal weight (EW) models

4 EWc

All cues are accorded equal weight (see, e.g., Einhorn \& Hogarth, 1975).

$5 \mathrm{EWb}$

All cues are accorded equal weight (see, e.g., Dawes, 1979),

\section{Hybrid models}

$6 \quad$ EW/DEBA

$7 \mathrm{EW} / \mathrm{SVb}$
Choose according to equal weights. If this results in a tie, use DEBA to resolve the choice (Hogarth \& Karelaia, in press).

Rank-order

Choose according to equal weights. If this results in a tie, resolve conflict by the single most important variable
Information

to consult

Rank-order

of

importance

of cues

None

All

The most important cue

Variable
Calculations

None

$(m-1)$
$k \frac{m(m-1)}{2}$
2

$m$ sums

$(m-1) \quad \frac{m(m-1)}{2}$

$m$ sums

$(m-1) \quad \frac{m(m-1)}{2}$

\section{Domran (DR) models}

8 DRc

If an alternative dominates, choose it. Otherwise, choose at random

between non-dominated alternatives.

$9 \mathrm{DRb}$

Same as DRc except based on binary variables.
None

None

$$
k(m-1) \quad k \frac{m(m-1)}{2}
$$

\section{Importance}

of cues
Well-known statistical model.

Same as MRc except based on binary variables.
$11 \mathrm{MRb}$

* For all models, the decision maker is assumed to know the sign of the zero order correlation between cues and the criterion. NOTE: $m=$ number of alternatives, $k=$ number of attributes, cues 
Table 2 - Key formulas for different models using continuous variables (for choosing best of three)

\begin{tabular}{|c|c|c|c|}
\hline & $\begin{array}{l}\text { Accuracy } \\
\underline{\text { conditions }}\end{array}$ & $\begin{array}{l}\underline{\text { Error }} \\
\underline{\text { variances }}\end{array}$ & $\frac{\text { Upper integration limits, }}{l_{a b} \text { and } l_{a c}}$ \\
\hline \multicolumn{4}{|l|}{ Single variable (SVc) } \\
\hline$Y_{a}=\rho_{y x} X_{a}+\varepsilon_{a}$ & $\rho_{y x}\left(X_{a}-X_{b}\right)>\varepsilon_{b}-\varepsilon_{a}$ & $2\left(1-\rho_{y x}^{2}\right)$ & $\frac{\rho_{y x}\left(x_{a}-x_{b}\right)}{\sqrt{2\left(1-\rho_{y x}^{2}\right)}}$ \\
\hline$Y_{b}=\rho_{y x} X_{b}+\varepsilon_{b}$ & $\rho_{y x}\left(X_{a}-X_{c}\right)>\varepsilon_{c}-\varepsilon_{a}$ & & $\frac{\rho_{y x}\left(x_{a}-x_{c}\right)}{\sqrt{2\left(1-\rho_{y x}^{2}\right)}}$ \\
\hline \multicolumn{4}{|l|}{$Y_{c}=\rho_{y x} X_{c}+\varepsilon_{c}$} \\
\hline \multirow{2}{*}{$Y_{a}=\left(\rho_{y \bar{x}} / \sigma_{\bar{x}}\right) \bar{X}_{a}+v_{a}$} & \multirow{2}{*}{$\left(\rho_{y \bar{x}} / \sigma_{\bar{x}}\right)\left(\bar{X}_{a}-\bar{X}_{b}\right)>v_{b}-v_{a}$} & \multirow{2}{*}{$2\left(1-\rho_{y \bar{x}}^{2}\right)$} & $\rho_{y \bar{x}}\left(\bar{x}_{a}-\bar{x}_{b}\right)$ \\
\hline & & & $\sigma_{\bar{x}} \sqrt{2\left(1-\rho_{y \bar{x}}^{2}\right)}$ \\
\hline \multirow{2}{*}{$Y_{b}=\left(\rho_{y \bar{x}} / \sigma_{\bar{x}}\right) \bar{X}_{b}+v_{b}$} & \multirow{2}{*}{$\left(\rho_{y \bar{x}} / \sigma_{\bar{x}}\right)\left(\bar{X}_{a}-\bar{X}_{c}\right)>v_{c}-v_{a}$} & & $\frac{\rho_{y \bar{x}}\left(\bar{x}_{a}-\bar{x}_{c}\right)}{}$ \\
\hline & & & $\sigma_{\bar{x}} \sqrt{2\left(1-\rho_{y \bar{x}}^{2}\right)}$ \\
\hline \multicolumn{4}{|l|}{$\begin{array}{l}\qquad y_{c}=\left(\rho_{y \bar{x}} / \sigma_{\bar{x}}\right) \bar{X}_{c}+v_{c} \\
\text { Multiple regression }(\mathrm{MRc})\end{array}$} \\
\hline \multirow{2}{*}{$Y_{a}=\hat{Y}_{a}+u_{a}$} & \multirow{2}{*}{$\left(\hat{Y}_{a}-\hat{Y}_{b}\right)>u_{b}-u_{a}$} & \multirow{2}{*}{$2\left(1-R_{a d j}^{2}\right)$} & $\left(\hat{y}_{a}-\hat{y}_{b}\right)$ \\
\hline & & & $\sqrt{2}\left(1-R_{a d j}^{2}\right)$ \\
\hline$Y_{b}=\hat{Y}_{b}+u_{b}$ & \multirow[t]{3}{*}{$\left(\hat{Y}_{a}-\hat{Y}_{c}\right)>u_{c}-u_{a}$} & & \\
\hline \multirow{2}{*}{$Y_{c}=\hat{Y}_{c}+u_{c}$} & & & $\left(\hat{y}_{a}-\hat{y}_{c}\right)$ \\
\hline & & & $\sqrt{2\left(1-R_{a d j}^{2}\right)}$ \\
\hline
\end{tabular}


Notes:

1. $\rho_{y \bar{x}}=\bar{\rho}_{y x} \sqrt{\frac{k}{1+(k-1) \bar{\rho}_{x_{i} x_{j}}}} \quad$ where $k=$ number of $x$ variables, $\bar{\rho}_{y x}=$ average correlation between $y$ and the $x$ 's, and

$$
\bar{\rho}_{x_{i} x_{j}}=\text { average inter-correlations amongst the } x \text { 's. }
$$

2. $\sigma_{\bar{x}}=\sqrt{\frac{1}{k}\left(1+(k-1) \bar{\rho}_{x_{i} x_{j}}\right)}$

3. $R_{a d j}^{2}=1-\left(1-R^{2}\right) \frac{(n-1)}{(n-k)} \quad$ where $n=$ number of observations.

4. The probability of optimal choice out of three is given by:

$$
\int_{-\infty-\infty}^{l_{a b} l_{a c}} \frac{1}{\pi \sqrt{3}} e^{-\frac{2}{3}\left(z_{1}^{2}-z_{1} z_{2}+z_{2}^{2}\right)} d z_{1} d z_{2}
$$

with upper integration limits being specific for different choice strategies.

5. Correlations between the standardized normal variables $z_{1}=\frac{\varepsilon_{b}-\varepsilon_{a}}{\sqrt{2\left(1-\rho_{y x}{ }^{2}\right.}}$ and $z_{2}=\frac{\varepsilon_{c}-\varepsilon_{a}}{\sqrt{2\left(1-\rho_{y x}^{2}\right)}}$ are all equal to $1 / 2-$ see Appendix A. 
$\underline{\text { Table } 3 \text { - Formulas for overall predictive accuracy of models using continuous variables }}$ (for choosing best of $m$ )

$\underline{\text { Model }}$

$V_{d}$

$\underline{\mathrm{SVc}}$

$$
\left(\begin{array}{ccc}
2 & \ldots & 1 \\
\cdots & \ldots & \ldots \\
1 & \ldots & 2
\end{array}\right)
$$

$\underline{\text { EWc }} \quad\left(\begin{array}{ccc}\sqrt{2} \sigma_{\bar{x}} & \ldots & \sigma_{\bar{x}}^{2} \\ \ldots & \ldots & \ldots \\ \sigma_{\bar{x}}^{2} & \ldots & \sqrt{2} \sigma_{\bar{x}}\end{array}\right)$

$\underline{\mathrm{MRc}} \quad\left(\begin{array}{ccc}\sqrt{2 R_{a d j}^{2}} & \cdots & R_{a d j}^{2} \\ \cdots & \cdots & \cdots \\ R_{a d j}^{2} & \cdots & \sqrt{2 R_{a d j}^{2}}\end{array}\right)$
Upper limits of integration

$$
d_{i}^{*}, \text { for } i=\overline{1, m-1}
$$

$$
\frac{\rho_{y x} d_{i}}{\sqrt{2\left(1-\rho_{y x}^{2}\right)}}
$$

$$
\frac{\rho_{y \bar{x}} d_{i}}{\sigma_{\bar{x}} \sqrt{2\left(1-\rho_{y \bar{x}}^{2}\right)}}
$$

$$
\frac{d_{i}}{\sqrt{2\left(1-R_{a d j}^{2}\right)}}
$$

\section{Notes:}

Overall expected probability of success is given by:

$$
m \int_{0}^{\infty} \ldots \int_{0}^{\infty} \varphi\left(d \mid \mu_{d}, V_{d}\right)\left[\int_{-\infty}^{d_{1}^{*} \ldots .} \int_{-\infty}^{d_{m-1}^{*}} \varphi\left(z \mid \mu_{z}, V_{z}\right) d z_{1} \ldots d z_{m-1}\right] d d_{1} \ldots d d_{m-1}
$$

where $z^{\prime}=\left(z_{1}, z_{2}, \ldots, z_{m-1}\right), d^{\prime}=\left(d_{1}, d_{2}, \ldots, d_{m-1}\right)$, and $V_{z}=\left(\begin{array}{ccc}1 & \ldots & 1 / 2 \\ \ldots & \ldots & \ldots \\ 1 / 2 & \ldots & 1\end{array}\right)$.

$V_{d}$ and $d_{i}^{*}$, for $i=\overline{1, m-1}$, are specific for different choice strategies.

See also notes to Table 2. 
Table 4 - Key formulas for different models using binary variables (for choosing best of three)

$\underline{\text { Model }}$

$\begin{array}{lccc}\underline{\text { Single variable }} & Y=\frac{\rho_{y w}}{\sigma_{w}} W+\varsigma & \frac{\rho_{y w}\left(w_{a}-w_{b}\right)}{\sigma_{w b} \sqrt{2\left(1-\rho_{y w}^{2}\right)}} & \frac{\rho_{y w}\left(w_{a}-w_{c}\right)}{\sigma_{w} \sqrt{2\left(1-\rho_{y w}^{2}\right)}} \\ \frac{\text { Equal weights }}{\underline{(\mathrm{EWb})}} & Y=\frac{\rho_{y \bar{w}}}{\sigma_{\bar{w}}} \bar{W}+\xi & \frac{\rho_{y \bar{w}}\left(\bar{w}_{a}-\bar{w}_{b}\right)}{\sigma_{\bar{w}} \sqrt{2\left(1-\rho_{y \bar{w}}^{2}\right)}} & \frac{\rho_{y \bar{w}}\left(\bar{w}_{a}-\bar{w}_{c}\right)}{\sigma_{\bar{w}} \sqrt{2\left(1-\rho_{y \bar{w}}^{2}\right)}} \\ \underline{\underline{\text { Multiple regression }}} & Y=\hat{Y}+\zeta & \frac{\left(\hat{y}_{a}-\hat{y}_{b}\right)}{\sqrt{2\left(1-R_{a d j}^{2}\right)}} & \frac{\left(\hat{y}_{a}-\hat{y}_{b}\right)}{\sqrt{2\left(1-R_{a d j}^{2}\right)}}\end{array}$

Notes:

1. $\rho_{y \bar{w}}=\bar{\rho}_{y w} \sqrt{\frac{k}{1+(k-1) \bar{\rho}_{w_{i} w_{j}}}}$,

where $k=$ number of $x$ variables,

$\bar{\rho}_{y w}=$ average correlation between $y$ and the $w$ 's, and

$\bar{\rho}_{w_{i} w_{j}}=$ average inter-correlations amongst the $w$ 's.

2. $\sigma_{\bar{w}}=\sqrt{\frac{1}{k}\left(1+(k-1) \bar{\rho}_{w_{i} w_{j}}\right)}$

3. $R_{a d j}^{2}=1-\left(1-R^{2}\right) \frac{(n-1)}{(n-k)}$

where $n=$ number of observations.

4. The probability of optimal choice out of three is given by

$$
\int_{-\infty-\infty}^{h_{a b} h_{a c}} \frac{1}{\pi \sqrt{3}} e^{-\frac{2}{3}\left(z_{1}^{2}-z_{1} z_{2}+z_{2}^{2}\right)} d z_{1} d z_{2},
$$

with upper integration limits being specific for different choice strategies. 
$\underline{\text { Table } 5 \text {-- Experimental design }}$

\begin{tabular}{|c|c|c|c|c|c|c|c|c|c|c|c|c|c|c|c|c|c|c|c|c|}
\hline \multirow[b]{3}{*}{$\begin{array}{l}\text { Variability of cue } \\
\text { validities: } \max \text { - min }\end{array}$} & \multicolumn{5}{|c|}{$\underline{\text { Case A }(k=3)}$} & \multicolumn{5}{|c|}{$\underline{\text { Case B }(\mathrm{k}=3)}$} & \multicolumn{5}{|c|}{$\underline{\text { Case } \mathrm{C}(\mathrm{k}=5)}$} & \multicolumn{5}{|c|}{$\underline{\text { Case D }(\mathrm{k}=5)}$} \\
\hline & $\underline{1}$ & $\underline{2}$ & $\underline{3}$ & $\underline{4}$ & $\underline{5}$ & $\underline{1}$ & $\underline{2}$ & $\underline{3}$ & $\underline{4}$ & $\underline{5}$ & $\underline{1}$ & $\underline{2}$ & $\underline{3}$ & $\underline{4}$ & $\underline{5}$ & $\underline{1}$ & $\underline{2}$ & $\underline{3}$ & $\underline{4}$ & $\underline{5}$ \\
\hline & 0.30 & 0.39 & 0.49 & 0.60 & 0.70 & 0.29 & 0.39 & 0.50 & 0.59 & 0.69 & 0.14 & 0.25 & 0.30 & 0.34 & 0.44 & 0.00 & 0.10 & 0.30 & 0.40 & 0.50 \\
\hline Validity of the first cue & 0.39 & 0.49 & 0.59 & 0.69 & 0.79 & 0.39 & 0.49 & 0.59 & 0.69 & 0.79 & 0.34 & 0.44 & 0.49 & 0.54 & 0.64 & 0.29 & 0.39 & 0.59 & 0.69 & 0.79 \\
\hline Average validity & 0.26 & 0.29 & 0.33 & 0.36 & 0.39 & 0.26 & 0.29 & 0.32 & 0.36 & 0.39 & 0.25 & 0.29 & 0.34 & 0.38 & 0.44 & 0.29 & 0.33 & 0.37 & 0.40 & 0.43 \\
\hline $\begin{array}{l}\text { Average intercorrel- } \\
\text { ation of x's }\end{array}$ & 0.00 & 0.00 & 0.00 & 0.00 & 0.00 & 0.49 & 0.49 & 0.49 & 0.49 & 0.49 & 0.10 & 0.10 & 0.10 & 0.10 & 0.10 & 0.60 & 0.60 & 0.60 & 0.60 & 0.60 \\
\hline Correlation: $\mathrm{y}$ and $\bar{x}$ & 0.45 & 0.51 & 0.57 & 0.63 & 0.68 & 0.31 & 0.36 & 0.39 & 0.44 & 0.48 & 0.47 & 0.56 & 0.65 & 0.73 & 0.84 & 0.35 & 0.40 & 0.45 & 0.48 & 0.52 \\
\hline$R^{2}(M R)$-- fit & 0.35 & 0.43 & 0.52 & 0.63 & 0.76 & 0.31 & 0.39 & 0.49 & 0.62 & 0.78 & 0.42 & 0.51 & 0.61 & 0.70 & 0.88 & 0.34 & 0.38 & 0.51 & 0.63 & 0.78 \\
\hline$(\mathrm{n}-1) /(\mathrm{n}-\mathrm{k})$ & 1.12 & 1.12 & 1.12 & 1.12 & 1.12 & 1.12 & 1.12 & 1.12 & 1.12 & 1.12 & 1.27 & 1.27 & 1.27 & 1.27 & 1.27 & 1.27 & 1.27 & 1.27 & 1.27 & 1.27 \\
\hline
\end{tabular}




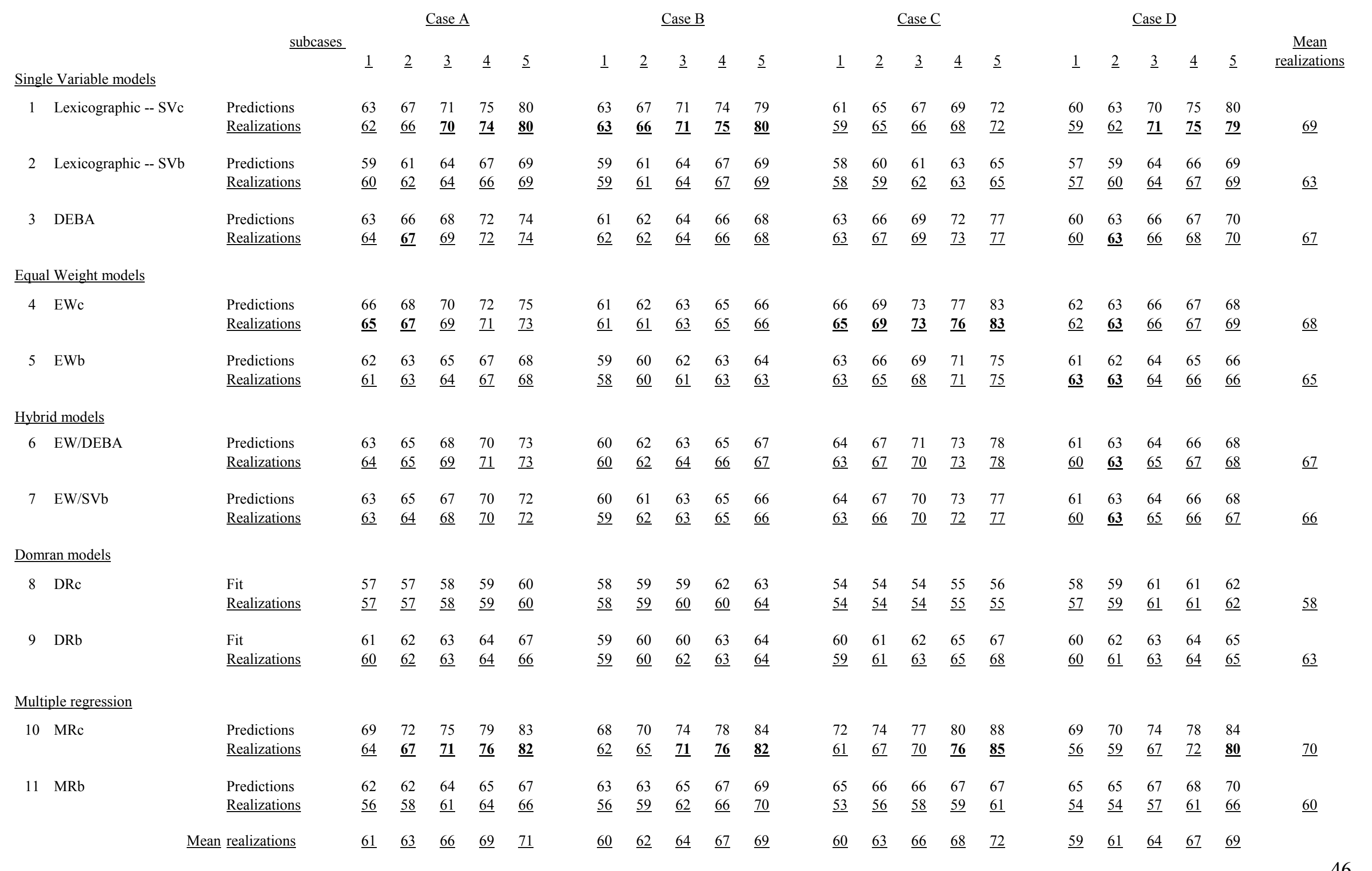


Table 7 -- Predictions and realizations (\% correct) for simulated data: Best of "three"

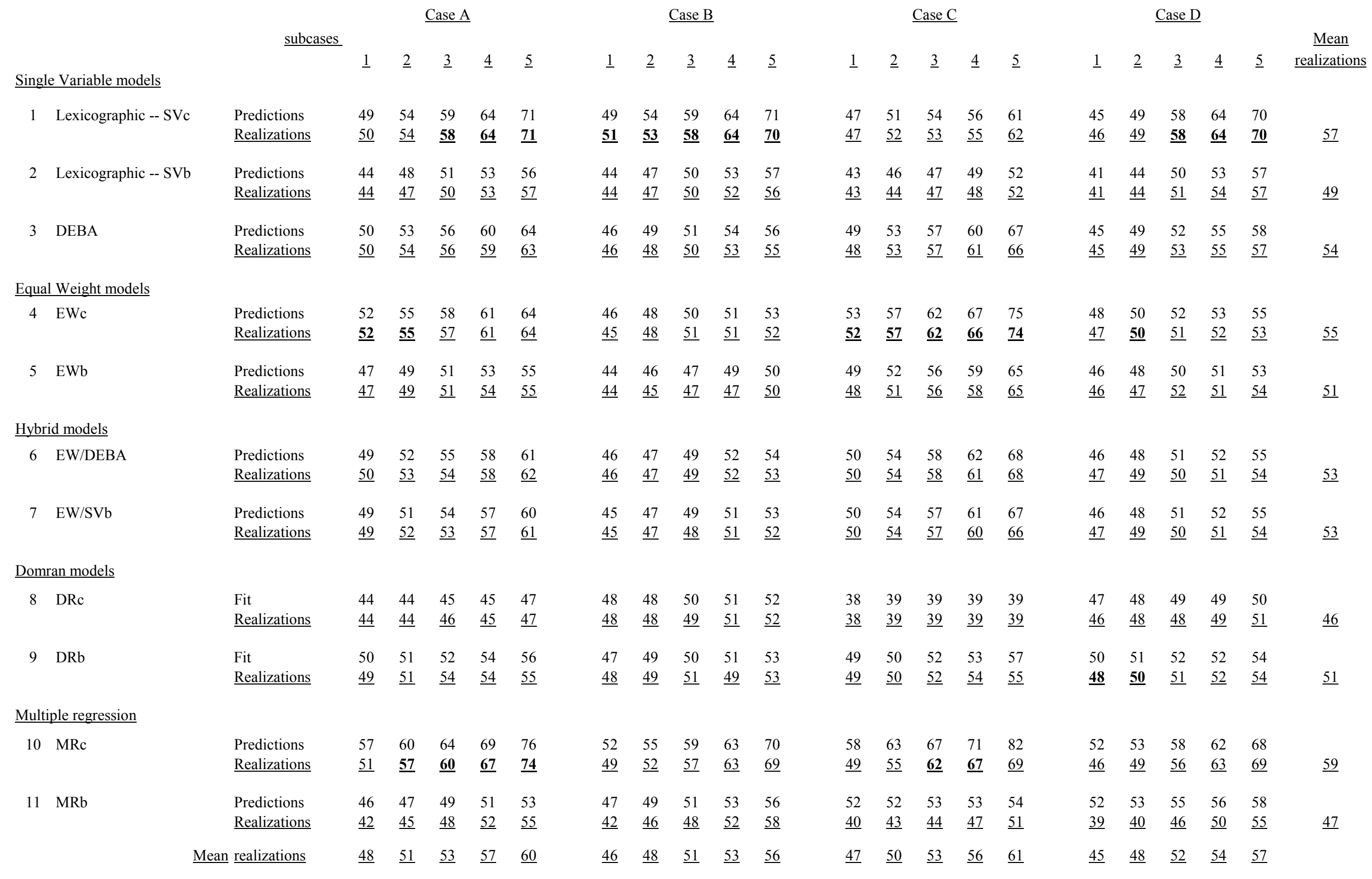

Note: Bold figures denote largest realization in each column or second largest if MRc is largest. 
$\underline{\text { Table } 8 \text {-- Predictions and realizations (\% correct) for simulated data: Best of "four" }}$

$\underline{\text { subcases }}$

Case A $\quad \underline{\text { Case B }}$ Case C

Single Variable models

1 Lexicographic -- SVc

2 Lexicographic -- SVb

3 DEBA

Equal Weight models

4 EWc

$5 \mathrm{EWb}$

Hybrid models

6 EW/DEBA

$7 \mathrm{EW} / \mathrm{SVb}$

Domran models

8 DRc

$9 \mathrm{DRb}$

Multiple regression

$10 \mathrm{MRc}$

$11 \mathrm{MRb}$

\begin{tabular}{|c|c|c|c|c|c|c|c|c|c|}
\hline Predictions & 50 & 54 & 58 & 63 & 71 & 44 & 47 & 52 & 57 \\
\hline$\underline{\text { Realizations }}$ & $\underline{45}$ & $\underline{48}$ & $\underline{54}$ & $\underline{60}$ & $\underline{69}$ & $\underline{40}$ & $\underline{44}$ & $\underline{51}$ & $\underline{57}$ \\
\hline Predictions & 38 & 39 & 41 & 43 & 45 & 39 & 41 & 43 & 45 \\
\hline$\underline{\text { Realizations }}$ & $\underline{33}$ & $\underline{36}$ & $\underline{39}$ & $\underline{42}$ & $\underline{45}$ & $\underline{34}$ & $\underline{37}$ & $\underline{40}$ & $\underline{45}$ \\
\hline Mean realizations & $\underline{40}$ & $\underline{43}$ & $\underline{45}$ & $\underline{49}$ & $\underline{53}$ & $\underline{39}$ & $\underline{40}$ & $\underline{43}$ & $\underline{46}$ \\
\hline
\end{tabular}

\section{$\begin{array}{lllll}1 & \underline{2} & \underline{3} & \underline{4} & \underline{5}\end{array}$}

\section{$\begin{array}{lllll}1 & 2 & \underline{3} & \underline{4} & \underline{5}\end{array}$}

Predictions

$\underline{\text { Realizations }}$

$\begin{array}{lllll}42 & 46 & 52 & 58 & 65 \\ \underline{41} & \underline{46} & \underline{\mathbf{5 2}} & \underline{\mathbf{5 6}} & \underline{\mathbf{6 5}}\end{array}$

$\begin{array}{lllll}41 & 46 & 52 & 58 & 65 \\ \underline{\mathbf{4 2}} & \underline{\mathbf{4 5}} & \underline{\mathbf{5 2}} & \underline{\mathbf{5 8}} & \underline{\mathbf{6 6}}\end{array}$

$\begin{array}{lllll}39 & 44 & 46 & 49 & 54\end{array}$

$\underline{38} \quad \underline{44} \quad \underline{45} \quad \underline{49} \quad \underline{55}$

\begin{tabular}{lllll}
34 & 37 & 38 & 40 & 43 \\
34 & 37 & 38 & 41 & 43 \\
\hline
\end{tabular}

Realization

$\begin{array}{lllll}35 & 38 & 41 & 44 & 47 \\ \underline{35} & \underline{38} & \underline{40} & \underline{44} & \underline{48}\end{array}$

\begin{tabular}{lllll}
35 & 38 & 41 & 44 & 47 \\
36 & 38 & 41 & 44 & 48 \\
\hline
\end{tabular}

Predictions

Realizations $\begin{array}{lllll}38 & 39 & 42 & 45 & 47 \\ \underline{39} & \underline{39} & \underline{42} & \underline{44} & \underline{46}\end{array}$

$\begin{array}{lllll}41 & 45 & 50 & 53 & 60 \\ 41 & \underline{46} & \underline{50} & \underline{54} & \underline{60}\end{array}$ $\underline{\text { Mean }} \underline{\text { realizations }}$

$\underline{40} \quad \underline{43} \quad \underline{45} \quad \underline{49} \quad \underline{53}$ $\underline{\text { Case D }}$

$\begin{array}{cccccc}\underline{1} & \underline{2} & \underline{3} & \underline{4} & \underline{5} & \begin{array}{c}\underline{\text { Mean }} \\ \text { realizations }\end{array} \\ 37 & 41 & 52 & 58 & 65 & \\ \underline{37} & \underline{41} & \underline{\mathbf{5 2}} & \underline{\mathbf{5 8}} & \underline{\mathbf{6 5}} & \underline{50} \\ 33 & 35 & 41 & 44 & 48 & \\ \underline{33} & \underline{34} & \underline{41} & \underline{44} & \underline{48} & \underline{40} \\ 37 & 39 & 44 & 45 & 49 & \\ \underline{38} & \underline{40} & \underline{44} & \underline{45} & \underline{49} & \underline{46}\end{array}$

$\begin{array}{lllll}40 & 42 & 44 & 46 & 48\end{array}$

$\underline{39} \quad \underline{42} \quad \underline{44} \quad \underline{44} \quad \underline{47}$

$\begin{array}{lllll}38 & 40 & 42 & 43 & 45\end{array}$

$\underline{37} \quad \underline{39} \quad \underline{42} \quad \underline{42} \quad \underline{45}$

\begin{tabular}{|c|c|c|c|c|c|c|c|c|c|}
\hline Predictions & 41 & 44 & 47 & 50 & 54 & 36 & 39 & 40 & 42 \\
\hline Realizations & $\underline{41}$ & $\underline{44}$ & $\underline{46}$ & $\underline{51}$ & $\underline{54}$ & $\underline{38}$ & $\underline{39}$ & $\underline{42}$ & $\underline{42}$ \\
\hline Predictions & 40 & 43 & 46 & 49 & 53 & 36 & 38 & 40 & 42 \\
\hline Realizations & $\underline{40}$ & $\underline{43}$ & $\underline{45}$ & $\underline{49}$ & $\underline{52}$ & $\underline{37}$ & $\underline{38}$ & $\underline{41}$ & $\underline{41}$ \\
\hline
\end{tabular}

$\begin{array}{lllll}43 & 47 & 51 & 56 & 62\end{array}$

$\underline{43} \quad \underline{47} \quad \underline{51} \quad \underline{55} \quad \underline{61}$

$\begin{array}{lllll}38 & 40 & 43 & 44 & 47\end{array}$

$\begin{array}{lllll}42 & 46 & 50 & 54 & 60\end{array}$

$\underline{42} \quad \underline{46} \quad \underline{50} \quad \underline{54} \quad \underline{59}$

$\underline{4} \quad \underline{42} \quad \underline{42} \quad \underline{47}$

$\begin{array}{lllll}38 & 40 & 42 & 43 & 46 \\ \underline{37} & \underline{41} & \underline{42} & \underline{42} & \underline{46}\end{array}$

$\underline{44}$

$\begin{array}{lllllllllll}\text { Fit } & 37 & 37 & 38 & 38 & 39 & 43 & 44 & 44 & 45 & 47\end{array}$

$\begin{array}{lllll}31 & 31 & 31 & 31 & 31\end{array}$

$\begin{array}{lllll}42 & 42 & 43 & 43 & 44\end{array}$

$\underline{41} \quad \underline{42} \quad \underline{43} \quad \underline{43} \quad \underline{44}$

$\begin{array}{lllll}44 & 45 & 47 & 49 & 51\end{array}$

$\begin{array}{lllll}44 & 45 & 47 & 46 & 47\end{array}$

$\underline{44} \quad \underline{45} \quad \underline{46} \quad \underline{47} \quad \underline{48}$

$\begin{array}{llllll}\text { Fit } & 45 & 45 & 47 & 48 & 49\end{array}$

$\begin{array}{lllll}42 & 44 & 44 & 45 & 48\end{array}$

$\underline{44} \quad \underline{45} \quad \underline{47} \quad \underline{48} \quad \underline{50}$

$\begin{array}{lllll}51 & 55 & 61 & 66 & 78\end{array}$

$\begin{array}{lllll}44 & 46 & 51 & 56 & 63\end{array}$

$\underline{38} \quad \underline{40} \quad \underline{50} \quad \underline{56} \quad \underline{\mathbf{6 5}}$

$\underline{53}$

$\begin{array}{lllll}45 & 45 & 46 & 46 & 48\end{array}$

$\begin{array}{lllll}45 & 45 & 48 & 50 & 52\end{array}$

$\underline{30} \quad \underline{30} \quad \underline{36} \quad \underline{39} \quad \underline{47}$

Note: Bold figures denote largest realization in each column or second largest if MRc is largest. 


\section{Table 9 -- Mapping relative success of different models: Realizations relative to MRc*}

$\underline{\text { subcases:** }} \quad \underline{1} \quad \underline{2} \quad \underline{3} \quad \underline{4} \quad \underline{5}$

No/low redundancy

$\begin{array}{lllll}1 & \underline{2} & \underline{3} & \underline{4} & \underline{5}\end{array}$

\begin{tabular}{|c|c|c|c|c|c|c|c|c|c|c|c|c|c|c|}
\hline \multicolumn{9}{|c|}{ No/low redundancy } & \multicolumn{6}{|c|}{ Moderate/high redundancy } \\
\hline \multirow[t]{4}{*}{ Case A } & $\mathrm{SVc}$ & 1 & 2 & 1 & 3 & 4 & Case B & $\mathrm{SVc}$ & $\underline{-2}$ & $\underline{-1}$ & $\underline{-1}$ & $\underline{-1}$ & $\underline{\mathbf{0}}$ & \\
\hline & DEBA & 1 & 2 & 4 & 8 & 11 & & DEBA & 3 & 5 & 7 & 10 & 15 & 3 cues/ \\
\hline & EWc & $\underline{-1}$ & 1 & 3 & 6 & 10 & & EWc & 4 & 5 & 7 & 11 & 18 & attributes \\
\hline & $\mathrm{DRb}$ & 2 & 6 & 6 & 13 & 19 & & $\mathrm{DRb}$ & 1 & 3 & 6 & 14 & 16 & \\
\hline \multirow[t]{5}{*}{ Case C } & $\mathrm{SVc}$ & 1 & $\underline{3}$ & 9 & 11 & 17 & $\underline{\text { Case D }}$ & $\mathrm{SVc}$ & $\underline{\mathbf{0}}$ & $\underline{\mathbf{0}}$ & $\underline{-3}$ & $\underline{-2}$ & $\underline{-1}$ & \\
\hline & DEBA & $\underline{1}$ & $\underline{2}$ & 5 & 6 & 14 & & DEBA & $\underline{1}$ & $\underline{\mathbf{0}}$ & 3 & 8 & 12 & 5 cues/ \\
\hline & EWc & $\underline{-3}$ & $\underline{-2}$ & $\underline{\mathbf{0}}$ & 1 & $\underline{5}$ & & EWc & $\underline{-1}$ & $\underline{-1}$ & 5 & 10 & 16 & attributes \\
\hline & $\mathrm{DRb}$ & $\underline{\mathbf{0}}$ & 5 & 10 & 13 & 24 & & $\mathrm{DRb}$ & $\underline{-2}$ & $\underline{-2}$ & 5 & 11 & 15 & \\
\hline & & $\begin{array}{l}\text { High } \\
\text { noise }\end{array}$ & & & & $\begin{array}{l}\text { Low } \\
\text { noise }\end{array}$ & & & $\begin{array}{l}\text { High } \\
\text { noise }\end{array}$ & & & & $\begin{array}{l}\text { Low } \\
\text { noise }\end{array}$ & \\
\hline
\end{tabular}

* Entries are performance (best of "three") of MRc less specified models.

Shaded regions indicate areas where MRc's advantage $>4 \%$; bold type shows no advantage or disadvantage of MRc.

** Parameters specifying the sub-cases are provided in Table 5 -- descriptions in bold. 
$\underline{\text { Table } 10 \text {-- Regressions of model performance (realizations) on environmental characteristics }}$

$\underline{\text { Models: }} \underline{\underline{\mathrm{SV}}} \quad \underline{\mathrm{SVb}} \quad \underline{\mathrm{DEBA}} \quad \underline{\mathrm{EWc}} \quad \underline{\mathrm{EWb}} \quad \underline{\underline{\mathrm{EW} /}} \quad \underline{\underline{\mathrm{EW}} /} \quad \underline{\mathrm{SVb}} \quad \underline{\mathrm{DRc}} \quad \underline{\mathrm{DRb}} \quad \underline{\mathrm{MRc}} \quad \underline{\mathrm{MRb}}$

$\underline{\text { Regression coefficients* for: }}$

Constant

Dummy1

Dummy2

Number of cues

Validity of the first cue

Correlation y and $\bar{x}$

Variability of cue validities: max - min

Average cue validity

Average intercorrelation of X's

$\mathrm{R}^{2}$

$\underline{\text { Regression statistics: }}$

$\mathrm{R}^{2}$

Estimated standard error

$42 \quad 47$

$-12 \quad-14$

$-7$

50

29

32

50

37

45

32

19

19

1.30

0.9

0.99

0.98

0.99

0.99

0.99

0.98

0.99

0.98

0.99

* All regression coefficients are statistically significant $(\mathrm{p}<.001)$. 
Table 11 -- Characteristics of empirical datasets

$\underline{\text { Golf rankings }} \quad \underline{\text { Golf earnings }} \quad \underline{\underline{\text { PhD programs }}} \quad \underline{\underline{\text { Consumer reports: }}}$

Variability of cue validities: $\max -\min$

Validity of the first cue

Average intercorrelation of $\mathrm{x}^{\prime} \mathrm{s}$

Correlation y and $\bar{x}$

$\mathrm{R}^{2}(\mathrm{MR})$-- fit

$(\mathrm{n}-1) /(\mathrm{n}-\mathrm{k})$
0.23

0.78

0.46

0.78

0.68

1.07
0.29

0.86

0.46

0.84

0.81

1.07
0.07

0.38

0.81

0.79

0.60

0.20

0.89

0.80

0.81

0.73

1.04

1.09 
Table 12 -- Predictions and realizations (\% correct) for empirical datasets

\begin{tabular}{|c|c|c|c|c|c|c|c|c|c|c|c|c|c|c|c|}
\hline \multirow{3}{*}{\multicolumn{2}{|c|}{$\underline{\text { Single Variable models }}$}} & & \multicolumn{3}{|c|}{$\frac{\text { Golf rankings }}{\text { Best of }}$} & \multicolumn{3}{|c|}{ Golf earnings } & \multicolumn{3}{|c|}{$\underline{\text { PhD programs }}$} & \multicolumn{3}{|c|}{ Digital cameras } & \multirow{2}{*}{$\stackrel{\text { Mean }}{\underline{\text { realizations }}}$} \\
\hline & & & $\underline{\text { Two }}$ & Three & $\underline{\text { Four }}$ & $\underline{\text { Two }}$ & Three & $\underline{\text { Four }}$ & $\underline{\text { Two }}$ & Three & $\underline{\text { Four }}$ & $\underline{\text { Two }}$ & Three & $\underline{\text { Four }}$ & \\
\hline & & & & & & & & & & & & & & & \\
\hline & Lexicographic -- SVc & Predictions & 79 & 69 & 62 & 82 & 77 & 74 & 77 & 71 & 69 & 74 & 64 & 59 & \\
\hline & & $\underline{\text { Realizations }}$ & $\underline{79}$ & $\underline{72}$ & $\underline{67}$ & $\underline{81}$ & $\underline{76}$ & $\underline{73}$ & $\underline{78}$ & $\underline{75}$ & $\underline{74}$ & $\underline{72}$ & $\underline{66}$ & $\underline{59}$ & $\underline{73}$ \\
\hline 2 & Lexicographic -- SVb & Predictions & 69 & 55 & 45 & 69 & 57 & 48 & 71 & 63 & 58 & 69 & 60 & 55 & \\
\hline & & $\underline{\text { Realizations }}$ & $\underline{69}$ & $\underline{56}$ & $\underline{46}$ & $\underline{73}$ & $\underline{61}$ & $\underline{53}$ & $\underline{71}$ & $\underline{63}$ & $\underline{57}$ & $\underline{69}$ & $\underline{64}$ & $\underline{58}$ & $\underline{62}$ \\
\hline 3 & DEBA & Predictions & 73 & 60 & 50 & 76 & 67 & 59 & 80 & 76 & 72 & 79 & 71 & 67 & \\
\hline & & $\underline{\text { Realizations }}$ & $\underline{73}$ & $\underline{60}$ & $\underline{49}$ & $\underline{77}$ & $\underline{71}$ & $\underline{65}$ & $\underline{80}$ & $\underline{76}$ & $\underline{74}$ & $\underline{79}$ & $\underline{73}$ & $\underline{67}$ & $\underline{70}$ \\
\hline Equi & 1 Weight models & & & & & & & & & & & & & & \\
\hline 4 & EWc & Predictions & 79 & 68 & 61 & 79 & 73 & 70 & 82 & 78 & 77 & 79 & 69 & 63 & \\
\hline & & $\underline{\text { Realizations }}$ & $\underline{77}$ & $\underline{68}$ & $\underline{62}$ & $\underline{77}$ & $\underline{72}$ & $\underline{68}$ & $\underline{86}$ & $\underline{83}$ & $\underline{81}$ & $\underline{76}$ & $\underline{72}$ & $\underline{65}$ & $\underline{74}$ \\
\hline 5 & EWb & Predictions & 71 & 57 & 49 & 75 & 66 & 61 & 79 & 74 & 71 & 77 & 69 & 65 & \\
\hline & & $\underline{\text { Realizations }}$ & $\underline{71}$ & $\underline{58}$ & $\underline{48}$ & $\underline{75}$ & $\underline{67}$ & $\underline{63}$ & $\underline{79}$ & $\underline{75}$ & $\underline{73}$ & $\underline{77}$ & $\underline{69}$ & $\underline{63}$ & $\underline{68}$ \\
\hline$\underline{\text { Hyb }}$ & id models & & & & & & & & & & & & & & \\
\hline 6 & EW/DEBA & Predictions & 72 & 59 & 50 & 76 & 67 & 62 & 79 & 76 & 73 & 78 & 69 & 67 & \\
\hline & & $\underline{\text { Realizations }}$ & $\underline{73}$ & $\underline{59}$ & $\underline{49}$ & $\underline{77}$ & $\underline{71}$ & $\underline{65}$ & $\underline{79}$ & $\underline{77}$ & $\underline{74}$ & $\underline{79}$ & $\underline{71}$ & $\underline{66}$ & $\underline{70}$ \\
\hline 7 & $\mathrm{EW} / \mathrm{SVb}$ & Predictions & 71 & 58 & 49 & 76 & 67 & 62 & 79 & 76 & 72 & 78 & 68 & 66 & \\
\hline & & $\underline{\text { Realizations }}$ & $\underline{72}$ & $\underline{58}$ & $\underline{49}$ & $\underline{78}$ & $\underline{71}$ & $\underline{65}$ & $\underline{79}$ & $\underline{76}$ & $\underline{74}$ & $\underline{79}$ & $\underline{71}$ & $\underline{66}$ & $\underline{70}$ \\
\hline Dom & ran models & & & & & & & & & & & & & & \\
\hline 8 & DRc & Fit & 69 & 58 & 51 & 70 & 62 & 58 & 77 & 70 & 66 & 74 & 63 & 57 & \\
\hline & & $\underline{\text { Realizations }}$ & $\underline{69}$ & $\underline{58}$ & $\underline{51}$ & $\underline{69}$ & $\underline{63}$ & $\underline{58}$ & $\underline{77}$ & $\underline{70}$ & $\underline{66}$ & $\underline{73}$ & $\underline{63}$ & $\underline{57}$ & $\underline{65}$ \\
\hline 9 & $\mathrm{DRb}$ & Fit & 70 & 60 & 53 & 74 & 65 & 60 & 78 & 75 & 73 & 75 & 65 & 58 & \\
\hline & & $\underline{\text { Realizations }}$ & $\underline{69}$ & $\underline{60}$ & $\underline{52}$ & $\underline{74}$ & $\underline{66}$ & $\underline{60}$ & $\underline{78}$ & $\underline{75}$ & $\underline{73}$ & $\underline{75}$ & $\underline{65}$ & $\underline{58}$ & $\underline{67}$ \\
\hline Mult & ple regression & & & & & & & & & & & & & & \\
\hline & MRc & Predictions & 81 & 70 & 63 & 83 & 79 & 77 & 82 & 78 & 78 & 81 & 73 & 67 & \\
\hline & & $\underline{\text { Realizations }}$ & $\underline{80}$ & $\underline{71}$ & $\underline{65}$ & $\underline{82}$ & $\underline{78}$ & $\underline{75}$ & $\underline{86}$ & $\underline{83}$ & $\underline{81}$ & $\underline{78}$ & $\underline{72}$ & $\underline{66}$ & $\underline{76}$ \\
\hline & $\mathrm{MRb}$ & Predictions & 64 & 51 & 42 & 64 & 51 & 44 & 72 & 64 & 60 & 66 & 54 & 48 & \\
\hline & & $\underline{\text { Realizations }}$ & $\underline{67}$ & $\underline{54}$ & $\underline{47}$ & $\underline{71}$ & $\underline{62}$ & $\underline{54}$ & $\underline{77}$ & $\underline{73}$ & $\underline{69}$ & $\underline{69}$ & $\underline{66}$ & $\underline{59}$ & $\underline{64}$ \\
\hline & & realizations & $\underline{73}$ & $\underline{61}$ & $\underline{53}$ & $\underline{76}$ & $\underline{69}$ & $\underline{63}$ & $\underline{79}$ & $\underline{75}$ & $\underline{72}$ & $\underline{75}$ & $\underline{68}$ & $\underline{62}$ & \\
\hline
\end{tabular}




\section{Figure 1}

(a)

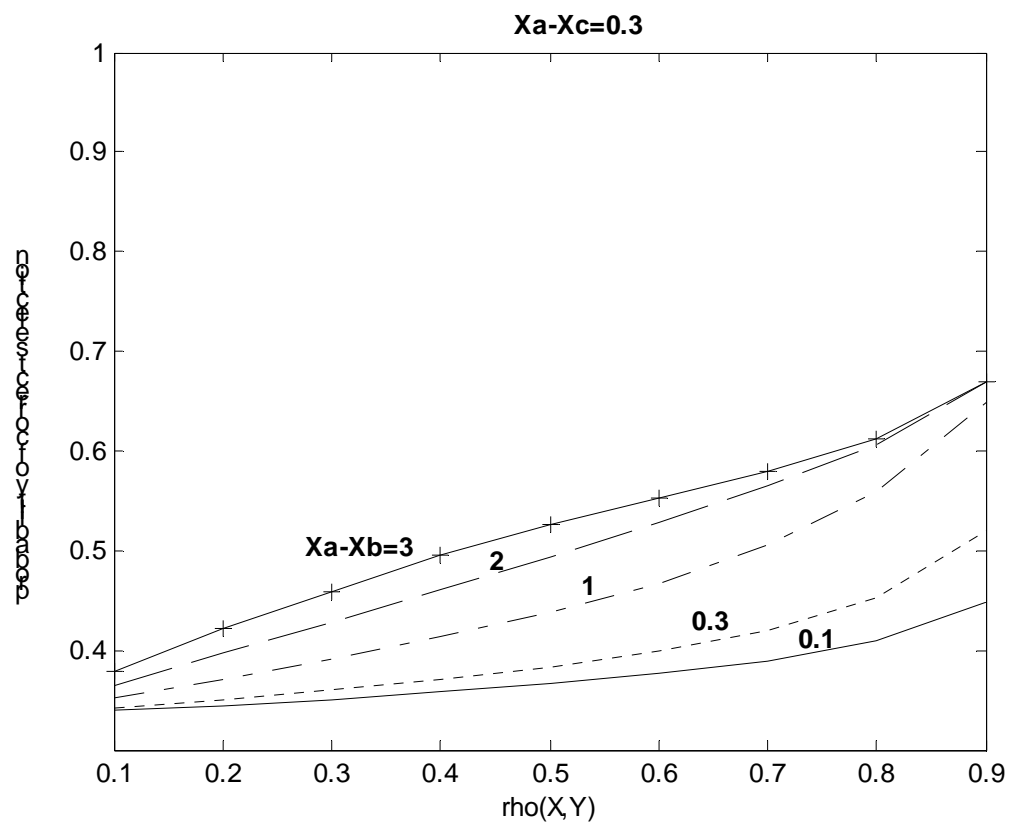

(b)

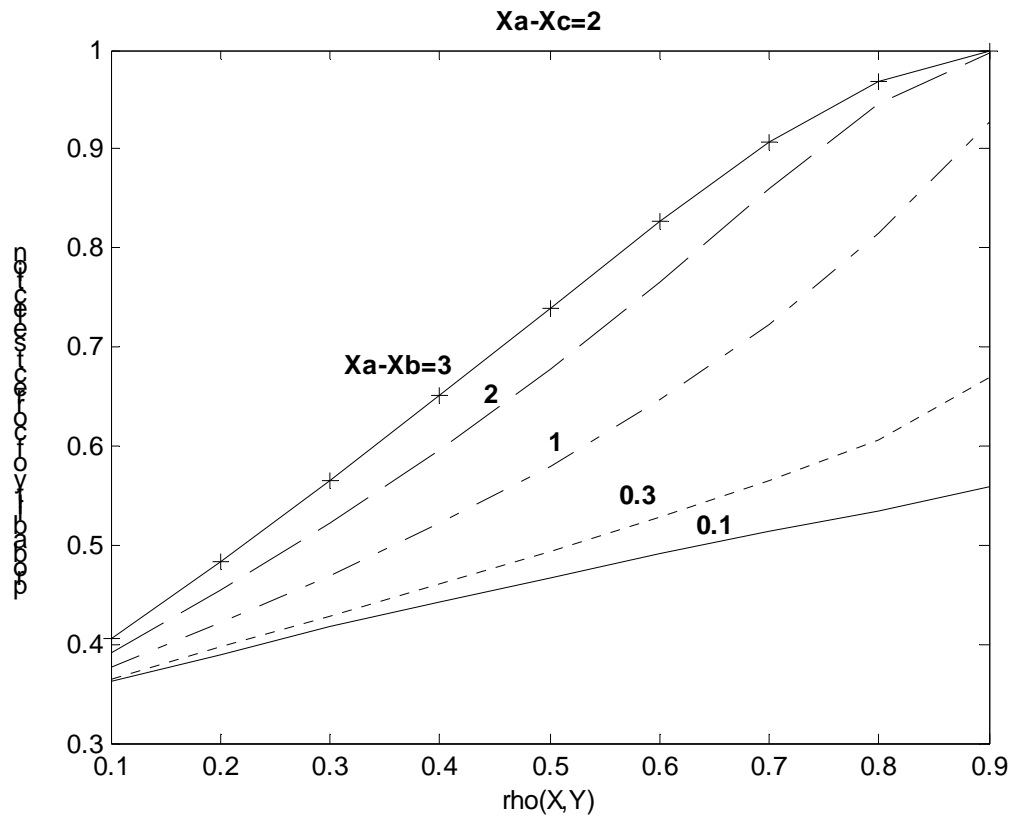


Figure 2

Percentage correct predictions by different models for conditions specified in Table 5 (cases A, B, C, and D): choosing one of three.
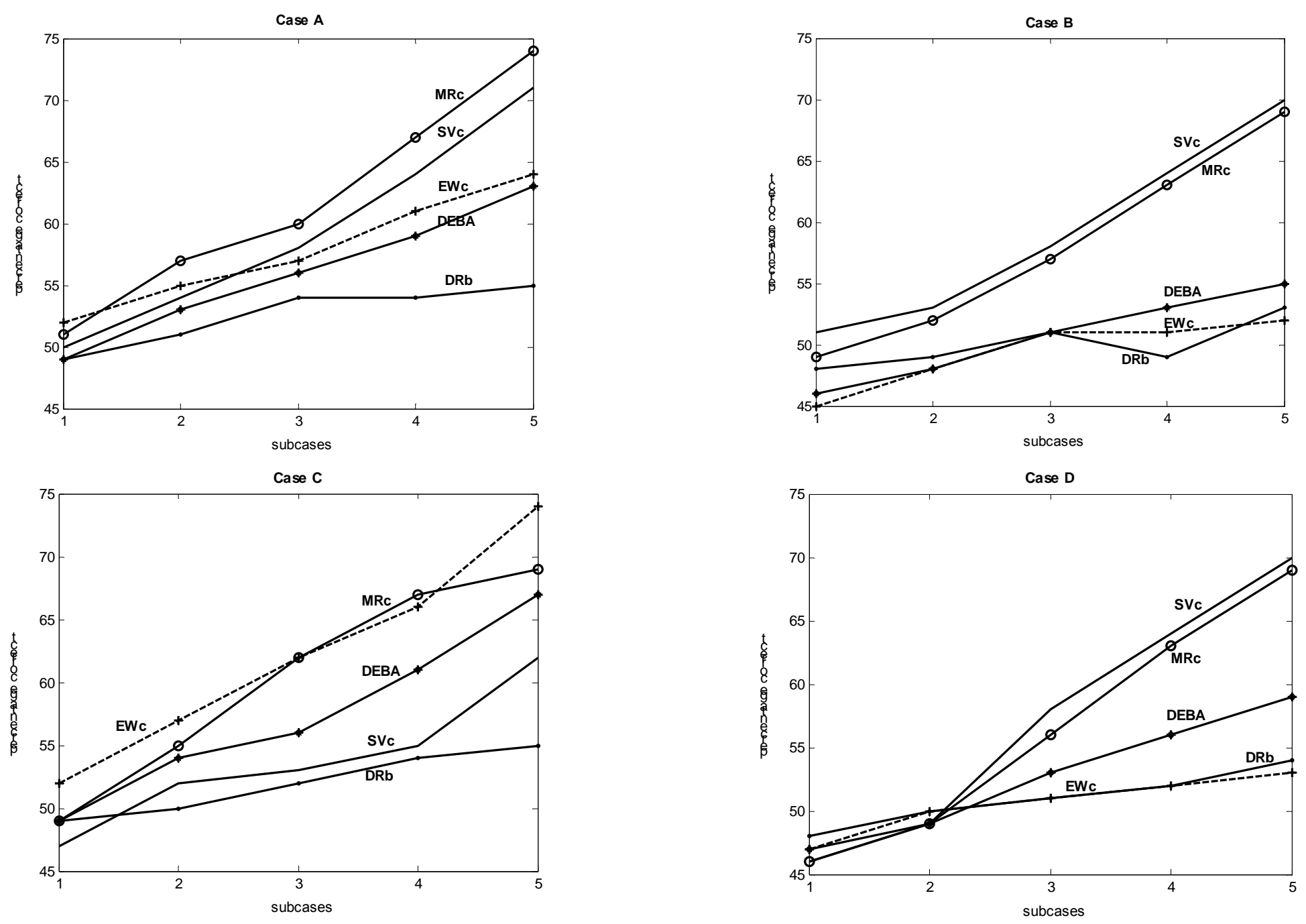
Figure 3

Percentage correct predictions by different models for real data sets specified in Table 12
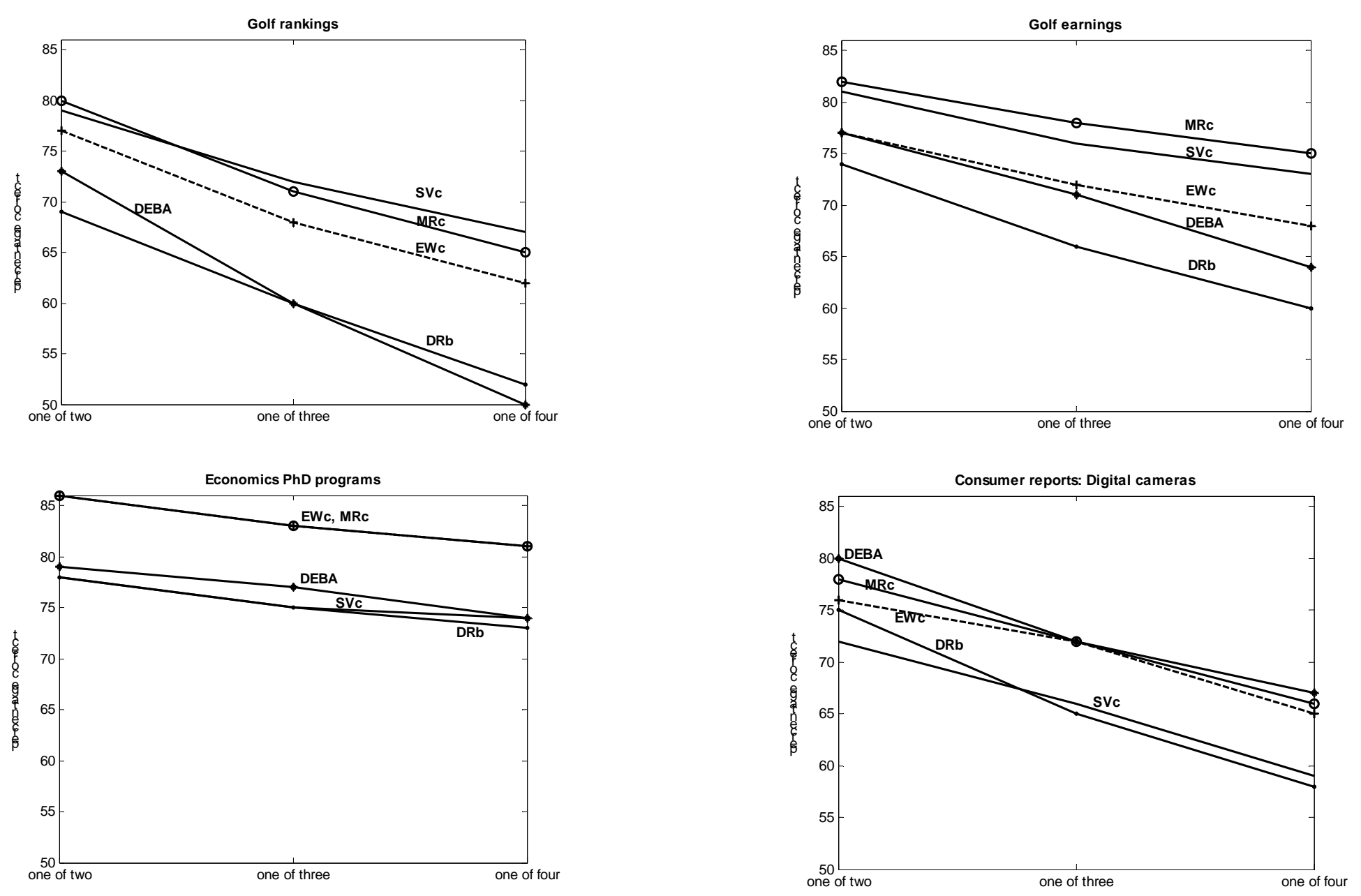
Appendix A - Correlation between $z_{1}=\frac{\varepsilon_{b}-\varepsilon_{a}}{\sqrt{2\left(1-\rho_{y x}{ }^{2}\right)}}$ and $z_{2}=\frac{\varepsilon_{c}-\varepsilon_{a}}{\sqrt{2\left(1-\rho_{y x}^{2}\right)}}$

Here we prove that $\rho_{z_{1}, z_{2}}=1 / 2$. First, by definition, and making use of the fact that $z_{1}$ and $z_{2}$ are standardized normal variables, we have:

$$
\begin{gathered}
\rho=\frac{\sigma_{z_{1}, z_{2}}}{\sigma_{z_{1}} \sigma_{z_{2}}}=\sigma_{z_{1}, z_{2}}= \\
\sigma\left(\frac{\varepsilon_{b}-\varepsilon_{a}}{\sqrt{2\left(1-\rho_{y x}^{2}\right)}}, \frac{\varepsilon_{c}-\varepsilon_{a}}{\sqrt{2\left(1-\rho_{y x}^{2}\right)}}\right)= \\
\frac{\sigma\left(\varepsilon_{b} \varepsilon_{c}-\varepsilon_{b} \varepsilon_{a}-\varepsilon_{a} \varepsilon_{c}+\varepsilon_{a} \varepsilon_{a}\right)}{2\left(1-\rho_{y x}^{2}\right)}
\end{gathered}
$$

Since $\varepsilon_{a}, \varepsilon_{b}$, and $\varepsilon_{c}$ are all independent of each other, (A3) can be re-expressed as

$$
\rho=\frac{\sigma^{2}\left(\varepsilon_{a}\right)}{2\left(1-\rho_{y x}^{2}\right)}=\frac{\left(1-\rho_{y x}^{2}\right)}{2\left(1-\rho_{y x}^{2}\right)}=\frac{1}{2}
$$

This result can also be generalized in the following way. First, imagine $m$ different alternatives each of which is characterized by a value of $X$, i.e., $x_{l}(l=1, \ldots, m)$ and an error term, $\varepsilon_{l}(l=1, \ldots m)$ in similar manner to equations (1) through (3) in the main text. Assume, without loss of generality, that $x_{1}$ is the largest $x$ value such that we want to calculate the probability that this alternative is better than all the others on the criterion. In this case, all pair-wise correlations between $z_{1 l}=\frac{\varepsilon_{l}-\varepsilon_{1}}{\sqrt{2\left(1-\rho_{y x}^{2}\right)}}$ and $z_{1 l+1}=\frac{\varepsilon_{l+1}-\varepsilon_{1}}{\sqrt{2\left(1-\rho_{y x}^{2}\right)}}$ for $l=2, \ldots . . m$, must - following the logic given above - all equal $1 / 2$. 


\section{Appendix B - Choosing the best of four: the single variable model (SV)}

Consider the case of four alternatives, A, B, C, and D with unknown values on the criterion variable, $Y$. Assume further that $\mathrm{A}$ is the preferred choice if $y_{a}>y_{b}$, $y_{a}>y_{c}$, and $y_{a}>y_{d}$. Without loss of generality, let alternative A have the largest value on a single attribute, $X$, i.e., $x_{a}>x_{b}, x_{a}>x_{c}$, and $x_{a}>x_{d}$. Given that it has the largest value, what is the probability that $\mathrm{A}$ is the optimal choice, i.e.,

$$
P\left\{\left(Y_{a}>Y_{b} \mid X_{a}=x_{a}>X_{b}=x_{b}\right) \cap\left(Y_{a}>Y_{c} \mid X_{a}=x_{a}>X_{c}=x_{c}\right) \cap\left(Y_{a}>Y_{d} \mid X_{a}=x_{a}>X_{d}=x_{d}\right)\right\} ?
$$

Analogically to the case of choice out of three alternatives, and making use of equation (12) in the main text, this probability can be found as

$$
\begin{aligned}
& P\left\{\left(\varepsilon_{b}-\varepsilon_{a}<\rho_{y x}\left(x_{a}-x_{b}\right)\right) \cap\left(\varepsilon_{c}-\varepsilon_{a}<\rho_{y x}\left(x_{a}-x_{c}\right)\right) \cap\left(\varepsilon_{d}-\varepsilon_{a}<\rho_{y x}\left(x_{d}-x_{c}\right)\right)\right\}= \\
& P\left\{\left(z_{1}<\frac{\rho_{y x}\left(x_{a}-x_{b}\right)}{\sqrt{2\left(1-\rho_{y x}^{2}\right)}}\right) \cap\left(z_{2}<\frac{\rho_{y x}\left(x_{a}-x_{c}\right)}{\sqrt{2\left(1-\rho_{y x}^{2}\right)}}\right) \cap\left(z_{3}<\frac{\rho_{y x}\left(x_{a}-x_{d}\right)}{\left.\sqrt{2\left(1-\rho_{y x}^{2}\right)}\right)}\right)\right\}
\end{aligned}
$$

where $z_{1}, z_{2}$, and $z_{3}$ are standardized normal variables, jointly distributed with all covariances being equal to $1 / 2$ (see Appendix A).

Applying the properties of the trivariate normal distribution (i.e., expression (12)), we find the target probability as:

$$
\begin{gathered}
\int_{-\infty-\infty-\infty}^{l_{a b} l_{a d} l_{a d}} \frac{1}{(2 \pi)^{3 / 2} \sqrt{1-\left(\rho_{12}^{2}+\rho_{13}^{2}+\rho_{23}^{2}\right)+2 \rho_{12} \rho_{13} \rho_{23}}} e^{-\frac{3}{4}\left(z_{1}^{2}+z_{2}^{2}+z_{3}^{2}\right)+\frac{1}{2}\left(z_{1} z_{2}+z_{1} z_{3}+z_{2} z_{3}\right)} d z_{1} d z_{2} d z_{3}= \\
\int_{-\infty-\infty-\infty}^{l_{a b} l_{a x} l_{a d}} \frac{1}{2 \pi^{3 / 2}} e^{-\frac{3}{4}\left(z_{1}^{2}+z_{2}^{2}+z_{3}^{2}\right)+\frac{1}{2}\left(z_{1} z_{2}+z_{1} z_{3}+z_{2} z_{3}\right)} d z_{1} d z_{2} d z_{3}
\end{gathered}
$$

where $\quad l_{a b}=\frac{\rho_{y x}\left(x_{a}-x_{b}\right)}{\sqrt{2\left(1-\rho_{y x}{ }^{2}\right)}} ; \quad l_{a c}=\frac{\rho_{y x}\left(x_{a}-x_{c}\right)}{\sqrt{2\left(1-\rho_{y x}{ }^{2}\right)}} ; \quad l_{a d}=\frac{\rho_{y x}\left(x_{a}-x_{d}\right)}{\sqrt{2\left(1-\rho_{y x}{ }^{2}\right)}}$. 